\title{
GCU
}

Glasgow Caledonian

University

University for the Common Good

\section{A virtual MIMO dual-hop architecture based on hybrid spatial modulation}

Stavridis, Athanasios; Basnayaka, Dushyantha; Sinanovic, Sinan; Di Renzo, Marco; Haas, Harald

Published in:

IEEE Transactions on Communications

DOI:

10.1109/TCOMM.2014.2343999

Publication date:

2014

Document Version

Author accepted manuscript

Link to publication in ResearchOnline

Citation for published version (Harvard):

Stavridis, A, Basnayaka, D, Sinanovic, S, Di Renzo, M \& Haas, H 2014, 'A virtual MIMO dual-hop architecture based on hybrid spatial modulation', IEEE Transactions on Communications, vol. 62, no. 9, pp. 3161-3179 . https://doi.org/10.1109/TCOMM.2014.2343999

\section{General rights}

Copyright and moral rights for the publications made accessible in the public portal are retained by the authors and/or other copyright owners and it is a condition of accessing publications that users recognise and abide by the legal requirements associated with these rights.

Take down policy

If you believe that this document breaches copyright please view our takedown policy at https://edshare.gcu.ac.uk/id/eprint/5179 for details of how to contact us. 


\title{
A Virtual MIMO Dual-Hop Architecture Based on Hybrid Spatial Modulation
}

\author{
Athanasios Stavridis, Student Member, IEEE, Dushyantha Basnayaka, Member, IEEE, \\ Sinan Sinanovic, Member, IEEE, Marco Di Renzo, Senior Member, IEEE, and Harald Haas, Member, IEEE
}

\begin{abstract}
In this paper, we propose a novel Virtual Multiple-Input-Multiple-Output (VMIMO) architecture based on the concept of Spatial Modulation (SM). Using a dual-hop and Decode-and-Forward protocol, we form a distributed system, called Dual-Hop Hybrid SM (DH-HSM). DH-HSM conveys information from a Source Node (SN) to a Destination Node (DN) via multiple Relay Nodes (RNs). The spatial position of the RNs is exploited for transferring information in addition to, or even without, a conventional symbol. In order to increase the performance of our architecture, while keeping the complexity of the RNs and DN low, we employ linear precoding using Channel State Information (CSI) at the SN. In this way, we form a Receive-Spatial Modulation (R-SM) pattern from the $\mathrm{SN}$ to the RNs, which is able to employ a centralized coordinated or a distributed uncoordinated detection algorithm at the RNs. In addition, we focus on the $\mathrm{SN}$ and propose two regularized linear precoding methods that employ realistic Imperfect Channel State Information at the Transmitter. The power of each precoder is analyzed theoretically. Using the Bit Error Rate (BER) metric, we evaluate our architecture against the following benchmark systems: 1) single relay; 2) best relay selection; 3) distributed Space Time Block Coding (STBC) VMIMO scheme; and 4) the direct communication link. We show that DH-HSM is able to achieve significant Signal-to-Noise Ratio (SNR) gains, which can be as high as $10.5 \mathrm{~dB}$ for a very large scale system setup. In order to verify our simulation results, we provide an analytical framework for the evaluation of the Average Bit Error Probability (ABEP).
\end{abstract}

Index Terms-MIMO, spatial modulation, linear precoding, relays, decode-and-forward.

Manuscript received December 18, 2013; revised April 30, 2014 and July 14, 2014; accepted July 19, 2014. Date of publication July 29, 2014; date of current version September 19, 2014. This work was supported by the European Union's Seventh Program for Research, Technological Development and Demonstration under Grant PITN-GA-2010-264759. This paper was presented in part at the IEEE 75th Vehicular Technology Conference (VTC2012-Spring), Yokohama, Japan, May, 2012 and at the 46th Asilomar Conference on Signals, Systems and Computers (ASILOMAR 2012), Pacific Grove, CA, USA, November, 2012. The associate editor coordinating the review of this paper and approving it for publication was J. Yuan.

A. Stavridis, D. Basnayaka, and H. Haas are with the Institute for Digital Communications and the Joint Research Institute for Signal and Image Processing, School of Engineering, The University of Edinburgh, Edinburgh EH9 3JL, U.K. (e-mail: a.stavridis@ed.ac.uk; d.basnayaka@ed.ac.uk; h.haas@ed.ac.uk).

S. Sinanovic is with the School of Engineering and Built Environment, Glasgow Caledonian University, Glasgow G4 0BA, U.K. (e-mail: Sinan. Sinanovic@gcu.ac.uk).

M. Di Renzo is with the Laboratory of Signals and Systems (L2S), French National Center for Scientific Research (CNRS), École Supérieure d'Électricité (SUPÉLEC), University of Paris-Sud XI, 91400 Paris, France (e-mail: marco.direnzo@1ss.supelec.fr).

\section{INTRODUCTION}

$\mathbf{O}$ VER the recent years Multiple-Input-Multiple-Output (MIMO) communication has attracted a tremendous attention from both the academia and industry. The deployment of multi-antenna elements, at both communicating ends, is considered to be an excellent candidate solution for the future spectrally efficient wireless networks. MIMO communication offers increased data rate without the need of any further radio resources [1].

Unfortunately, MIMO comes with the major disadvantage of increased hardware complexity. Multi-antenna elements require multiple Radio Frequency (RF) chains (usually equal to the number of the employed antennas). In addition, most of the time, optimum Maximum Likelihood (ML) detection at the receiver has an exponential complexity increase with the number of receive (or transmit) antennas [2]. Other major drawbacks of MIMO are the high energy consumption due to the increased number of RF chains [3] and the complex signal processing techniques.

In order to overcome the previous native problems of MIMO, research has focused on Virtual MIMO (VMIMO) systems. The main rationale behind VMIMO is to promote low complexity system implementation by combining MIMO and relay techniques. Due to the broad nature of VMIMO, there is a wide range of designs proposed in the literature. From the MIMO point of view, there are designs that achieve diversity gains [4], multiplexing gains [5], beamforming gains [6], or a combination of them. Moreover, from the relays' point of view, there are several communication protocols. The most famous protocols are Amplify-and-Forward (AF) [6], Decodeand-Forward (DF) [4], Compress-and-Forward (CF) [7], and Estimate-and-Forward (EF) [8]. Furthermore, the previous protocols are applied in two [9] or multiple hops [4].

\section{A. Related Work and Motivation}

A new open-loop MIMO scheme that enjoys a single RF chain configuration at the transmitter and successfully treats the inherent problems of MIMO is Spatial Modulation (SM) [10][12]. SM is a single RF chain scheme, which is able to employ an optimum single-stream ML detector. In following we summarize the main advantages of SM: i) SM is able to achieve a multiplexing gain with the activation of a single transmit antenna; ii) SM does not require Inter-Antenna Synchronization (IAS) at the transmitter; iii) the complexity of the transmitter 
does not scale significantly with the increase of antennas; and iv) SM offers increased energy efficiency. Especially, in terms of energy efficiency (bits/J), [3] show that the single RF chain configuration of SM results into significant benefits. We note that an important low-complexity and low-rate variation of SM, called Space Shift Keying (SSK) is proposed in [13]. The first real system implementation of SM is presented in [14]. Furthermore, the performance of SM under real channel measurements is performed in [15], [16]. A complete introduction on SM is provided in [17].

Until recently, MIMO precoding using Channel State Information at the Transmitter (CSIT) has been used either for the simplification of the receiver and receive power consuming benefits, or capacity gains, or the formation of Space Division Multiple Access (SDMA) schemes. In [18], MIMO precoding is used in a totally different way. Using Zero Forcing (ZF) or Minimum Mean Square Error (MMSE) precoding and the appropriate signal formation, a SM-like scheme is formed. For reasons of simplicity and in agreement with [19], we use the name of Receive-Spatial Modulation (R-SM) for this scheme. R-SM applies the space modulation concept at the receiver side.

Over the recent years, there have been many schemes that extend the concept of SM/SSK to a VMIMO distributed scenario, both for the AF and DF protocols. The authors of [20] study the performance analysis of a SSK-based AF relay network. In [21], using the idea of SSK, a cooperative transmission scheme is considered, both for the AF and DF protocols. The generalization of SSK in a two-way AF relays network is conducted in [22]. The adoption of SSK in a DF cooperative system is considered in [23]. Furthermore, the extension of SSK to a multi-branch and multi-hop scenario is studied in [24]. Moreover, the extension of Space Time Shift Keying (STSK) [25], which is a novel SM-like scheme, in a distributed VMIMO scenario is conducted in [26].

In [27], multi-antenna elements are deployed in such a way that form a dual hop scheme based on SM. The application of distributed SM to the uplink is studied in [28]. Also, [29] proposes a SSK scheme again for the uplink. In [30], using the ergodic capacity and the outage probability, it is shown that space modulation VMIMO schemes are able to achieve high throughput. Another extension of SM in a low complexity cooperative DF VMIMO architecture is conducted in [31]. The integration of Space Time Block Coding (STBC) in a distributed SM system is done in [32].

Unfortunately, all distributed and uncoded space modulation VMIMO schemes that employ multiple uncoordinated Relay Nodes (RNs) suffer from the erroneous activation of multiple RNs during the relaying phase. Thus, a Bit Error Rate (BER) performance degradation occurs. In order to overcome this problem, three strategies are followed in the literature: i) coordination between the RNs in order to ensure the activation of a single RN [33] (which is similar to the scenario of a single RN with multiple antennas [27]); ii) block-based transmission which allows the use of error correction codes at the RNs [21], [24]; and iii) advanced and error-aware detection techniques at the Destination Node (DN), which usually results in high complexity [28], [32].

\section{B. Contributions and Outcomes}

Against this background, we aim to propose and study an architecture which is suitable for the downlink. Motivated by the concept of VMIMO and the potential of SM/R-SM, we propose a non-cooperative relay architecture based on SM/ R-SM. Using a half-duplex DF protocol we propose a scheme that achieves information conveyance using R-SM [19] in the first hop and SM [12] in the second hop. In this way, a DualHop Hybrid Spatial Modulation (DH-HSM) system is formed. The term Hybrid Spatial Modulation (HSM) stems from the fact that our architecture employs R-SM in the first hop and SM in the second hop. The RNs are able to employ a Centralized Detection (CD) or a Distributed Detection (DD) algorithm, depending on their ability to coordinate. DH-HSM conveys information by extending the novel transmission mechanism of SM in a distributed framework.

In order to practically solve or reduce the problem of the activation of multiple RNs, without affecting the complexity of the RNs or the DN, we carefully design the transmission mode of the first hop. We use MIMO linear precoding with CSIT. In this way, we practically eliminate or reduce the effect of the wireless channel and offer significant receive Signal-to-Noise Ratio (SNR) gains at the RNs.

We consider realistic Imperfect-CSIT (I-CSIT) at the Source Node (SN) and propose two regularized linear precoding methods. The first precoder is obtained using statistical I-CSIT, while the second precoder employs worst-case I-CSIT. Furthermore, using an analytical framework, we study the instantaneous and the average power of all precoders used in this paper.

The evaluation of DH-HSM against the State-of-the-Art (SotA) is conducted using the metric of the Bit Error Rate (BER). The benchmark systems that we employ are the: i) single relay, ii) best relay selection, and iii) distributed STBC dual hop architectures, and iv) the direct communication link. Even though the comparison of DH-HSM with the best relay selection and distributed STBC is not fair for DH-HSM, our architecture is able to achieve significant BER gains in the majority of the system setups. We show that the gain of $\mathrm{DH}$ HSM over the best relay selection scheme can reach up to $9 \mathrm{~dB}$ for $\mathrm{BER}=10^{-4}$ and a very large scale system setup. Finally, in order to validate the obtained simulation results, we analyze the Average Bit Error Probability (ABEP) using the well known union bound method [34]. We emphasize that the derivation of the ABEP is conducted both for the CD and DD algorithms at the RNs.

The rest of the paper is organized as follows: Section II presents the system model of the proposed architecture when the RNs employ CD. Section III concentrates at the SN and proposes two precoding methods that take into account I-CSIT at the $\mathrm{SN}$. In addition, the same section provides a mathematical analysis of the instantaneous and average transmit power of the SN. In order to avoid coordination between the RNs, in Section IV we propose a DD algorithm which can be independently executed by the RNs. The derivation of the ABEP of our architecture is presented in Section V. Section VI provides results that evaluate the performance of the proposed scheme and precoding methods. Finally, we conclude this paper in Section VII. 


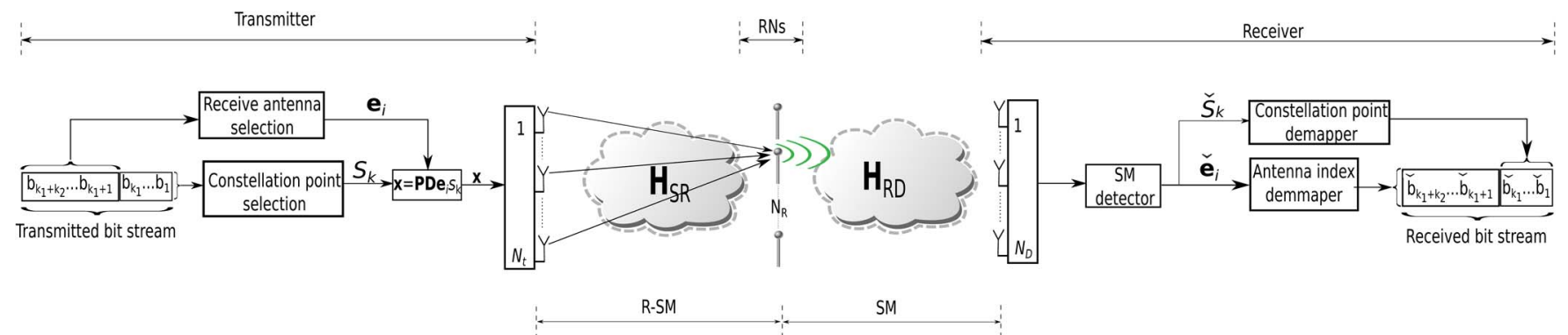

Fig. 1. System model of DH-HSM.

Notation: In the following, lowercase bold letters denote vectors and uppercase bolt letters denote matrices. $(\cdot)^{T},(\cdot)^{H}$, $\operatorname{tr}(\cdot)$ and $\mathbf{A}^{1 / 2}$ denote transpose, Hermitian transpose, matrix trace and the square root of $\mathbf{A}$, respectively. The Kronecker product is denoted as $\otimes$. $\|\cdot\|_{2}$ represents the Euclidean norm, while $\|\cdot\|_{F}$ stands for the Frobenius norm. $\operatorname{diag}\left(a_{1}, \ldots, a_{n}\right)$ represents a diagonal matrix whose main diagonal includes the elements $a_{1}, \ldots, a_{n} . \mathrm{E}[\cdot]$ is the mean value of a Random Variable (RV). A complex Gaussian distribution with mean $m$ and variance $\sigma_{C}^{2}$ is represented as $\mathcal{C N}\left(m, \sigma_{C}^{2}\right)$, where its real and imaginary part are independent and identically distributed (i.i.d.) Gaussian $\mathrm{RV}$ with distribution $\mathcal{N}\left(m,\left(\sigma_{C}^{2} / 2\right)\right) . \operatorname{Re}\{\cdot\}$ denotes the real part of a complex number or matrix.

\section{SySTEM MODEL}

Fig. 1 shows the system model of the proposed DH-HSM architecture. We consider a Base Station (BS) equipped with $N_{t}$ antennas which acts as the SN. Furthermore, we assume that our architecture includes $N_{R}$ single antenna RNs connected with an error-free backhaul link. Later in Section IV, we extend our architecture in a distributed framework where there is no backhaul connection between the RNs. The DN can be considered as a single or multi-antenna node (with $N_{D}$ antennas).

In addition, we assume that there is no direct link between the $\mathrm{SN}$ and the DN due to the poor channel condition. The SN acquires CSIT using either the reciprocity principle when it is applicable or using a low-rate feedback link from the relays. The DN acquires CSI using a training sequence transmitted from the RNs. Especially, when Time Division Duplexing (TDD) is employed, DH-HSM is able to supply the SN and the DN with Channel State Information (CSI) by transmitting a single training sequence from the RNs. In this section, we assume Perfect-CSIT (P-CSIT) at DN for the purpose of system presentation and in Section III we modify our architecture in order to operate under different practical scenarios of I-CSIT at the SN.

We configure our system with $N_{t} \geq N_{R}$ and $N_{R}$ equal to a power of 2. Under the assumptions that the transmitter employs a linear precoder and the wireless channel is flat quasi-static, the system equation of the first hop is expressed in a matrix form as:

$$
\mathbf{y}_{\mathrm{R}}=\mathbf{H}_{\mathrm{SR}} \mathbf{P D x}+\mathbf{w}_{\mathrm{SR}} .
$$

In (1), $\mathbf{y}_{\mathrm{R}}$ represents the $N_{R} \times 1$ received signal at the RNs in vector form. $\mathbf{H}_{\mathrm{SR}} \in \mathcal{C}^{N_{R} \times N_{t}}$ represents the channel matrix which is assumed to distribute as $\mathbf{H}_{\mathrm{SR}} \sim \mathcal{C N}(\mathbf{0}, \mathbf{I})$. The $N_{t} \times$ $N_{R}$ linear precoder is denoted as $\mathbf{P}$. $\mathbf{D}$ is a $N_{R} \times N_{R}$ diagonal matrix which is used to ensure that the transmitted power is not amplified by the precoder. The $i$-th element of the main diagonal of $\mathbf{D}$ is equal to $d_{i}=\sqrt{1 /\left\|\mathbf{p}_{i}\right\|_{2}^{2}}$, where $\mathbf{p}_{i}$ is the $i$ th column of $\mathbf{P}$. In this way, each column of the normalized precoder $\mathbf{P}_{\mathrm{n}}=\mathbf{P D}$ is constrained to have unity power. Furthermore, $\mathbf{x}$ represents the transmitting symbol vector. In this paper, we set the elements of $\mathbf{x}$ such that $\mathrm{E}_{\mathbf{x}}\left[\|\mathbf{x}\|_{2}^{2}\right]=1$. Finally, $\mathbf{w}_{S R} \in \mathcal{C}^{N_{D}}$ denotes the i.i.d. additive complex Gaussian noise with $\mathbf{w}_{S R_{i}} \sim \mathcal{C N}\left(0, \sigma_{w_{S R}}^{2}\right)$. We note that due to the use of the linear precoder $\mathbf{P}$, the DN requires multiple $\left(N_{t}\right) \mathrm{RF}$ chains.

In this paper, under the assumption of P-CSIT at the SN, we focus on two types of linear precoding: i) $\mathrm{ZF}$ and ii) MMSE. In traditional MIMO schemes, ZF (Bezout) precoding is a suboptimal method that offers a good trade-off between complexity and performance [35]. The main characteristic of $\mathrm{ZF}$ is the total elimination of the Inter-Channel Interference (ICI) at the receiver $\left(\mathbf{H}_{\mathrm{SR}} \mathbf{P}_{\mathrm{ZF}}=\mathbf{I}_{N_{R}, N_{R}}\right)$. The ZF precoding matrix is just the pseudo-inverse of the channel matrix $\mathbf{H}_{\mathrm{SR}}$. Using the Singular Value Decomposition (SVD), the precoding matrix is equal to

$$
\mathbf{P}_{\mathrm{ZF}}=\mathbf{V} \boldsymbol{\Sigma}^{-1} \mathbf{U}^{H},
$$

where $\mathbf{H}_{\mathrm{SR}}=\mathbf{U} \boldsymbol{\Sigma} \mathbf{V}^{H}$. Both $\mathbf{U}$ and $\mathbf{V}$ are unitary matrices with size $N_{R} \times N_{R}$ and $N_{t} \times N_{t}$, respectively. $\boldsymbol{\Sigma}$ is a $N_{R} \times N_{t}$ diagonal matrix whose main diagonal represents the $r$ real singular values $\sigma_{i}$ of $\mathbf{H}_{\mathrm{SR}}$. Here, $r$ denotes the rank of the channel matrix $\mathbf{H}_{\mathrm{SR}}$. A different way to express the $\mathrm{ZF}$ precoder is given below:

$$
\mathbf{P}_{\mathrm{ZF}}=\mathbf{H}_{\mathrm{SR}}^{H}\left(\mathbf{H}_{\mathrm{SR}} \mathbf{H}_{\mathrm{SR}}^{H}\right)^{-1} .
$$

An alternative method, suitable for rank-deficient channel matrices, is MMSE (Regularized ZF) precoding. The MMSE is given as:

$$
\mathbf{P}_{\mathrm{MMSE}}=\mathbf{H}_{\mathrm{SR}}^{H}\left[\mathbf{H}_{\mathrm{SR}} \mathbf{H}_{\mathrm{SR}}^{H}+\mu \mathbf{I}_{N_{R}, N_{R}}\right]^{-1},
$$

where $\mu$ is the regularization factor. Usually, $\mu$ is defined as $\mu=N_{t} \sigma_{w}^{2} / P_{\mathbf{s}}=N_{t} /$ SNR [36]. In traditional MIMO, MMSE precoding outperforms ZF precoding in low SNRs and approaches the ZF performance in high SNRs.

In order to form a R-SM transmission mechanism from the SN to the RNs, such as [18], [19], we set the transmitted signal vector $\mathbf{x} \widehat{=} \mathbf{x}\left(i, s_{k}\right)=\mathbf{e}_{i} s_{k}$. Where $\mathbf{e}_{i}=[0, \ldots, 0,1,0, \ldots, 0]^{T}$, 
$i=1, \ldots, N_{R}$, is a $N_{R} \times 1$ vector, with all of its elements equal to zero except the $i$-th element which is equal to 1 . The value of $i$ corresponds to the index of the receiving $\mathrm{RN}$. The indices $i$ are allocated to the RNs in the Medium Access Layer (MAC). $s_{k} \in\left\{s_{1}, \ldots, s_{M}\right\}$ denotes the transmitted symbol selected from a conventional constellation. Hence, depending on the bits to be transmitted, $k_{1}=\log _{2} N_{R}$ bits are used for the selection of $i$ and $k_{2}=\log _{2} M$ bits are encoded using the constellation point $s_{k}$. In this way, $k_{1}+k_{2}$ bits are transmitted from the $\mathrm{SN}$ to the RNs in a symbol period (first hop).

Under the assumption of ZF precoding using P-CSIT at the $\mathrm{SN}$, the observation at each of the RNs is given as:

$$
\mathbf{y}_{R}=\mathbf{D} \mathbf{x}+\mathbf{w}_{S R}
$$

If we take into account the sparsity of $\mathbf{x},(5)$ is written as:

$$
\begin{array}{ll}
y_{R_{j}}=d_{j} s_{k}+w_{S R_{j}}, & j=i, \\
y_{R_{j}}=w_{S R_{j}}, & j \neq i,
\end{array}
$$

where $j=1, \ldots, N_{R}$.

Due to the fact that the RNs communicate via a backhaullink, we are able to employ the following centralized lowcomplexity ML detector:

$$
\left(\hat{i}, \hat{s}_{k}\right)=\arg \min _{i, s_{k}} d_{i}^{2}\left|s_{k}\right|^{2}-2 d_{i} \operatorname{Re}\left\{y_{R_{i}^{*}} s_{k}\right\}
$$

As we can see from (7), the CSI that the R-SM detector requires is $d_{i}, i=1, \ldots, N_{R}$. We note that (7) ensures the activation of a single RN during the second hop.

During the second slot, we apply a SM-like transmission at which only the receiving $\mathrm{RN}$ from the previous slot is activated. This RN acts as a single antenna in a conventional co-located SM system. In this way, the system equation of the RNs-DN link is given as:

$$
\mathbf{y}_{D}=\mathbf{H}_{R D} \mathbf{x}\left(\hat{i}, \hat{s}_{k}\right)+\mathbf{w}_{R D}
$$

where $\mathbf{H}_{\mathrm{RD}} \sim \mathcal{C N}(\mathbf{0}, \mathbf{I})$ stands for the $N_{D} \times N_{R}$ RNs-DN wireless channel. $\mathbf{x}\left(\hat{i}, \hat{s}_{k}\right)=\mathbf{e}_{\hat{i}} \hat{s}_{k}$ is the $N_{R} \times 1$ vectorized detected symbol from the previous slot. Moreover, $\mathbf{w}_{R D} \in$ $\mathcal{C}^{N_{D}}$ is the i.i.d. additive complex Gaussian noise with $\mathbf{w}_{R D_{i}} \sim$ $\mathcal{C N}\left(0, \sigma_{w_{R D}}^{2}\right)$. Finally, $\mathbf{y}_{D}$ stands for the received signal at the DN. The optimal ML detection for SM is given in [11]

$$
\left(\check{i}, \check{s}_{k}\right)=\arg \min _{i, s_{k}}\left\|\mathbf{y}_{D}-\mathbf{H}_{R D} \mathbf{e}_{i} s_{k}\right\|_{2}^{2} \text {. }
$$

Hence, in this way, we form a dual-hop architecture which conveys $k_{t}=\left(k_{1}+k_{2}\right) / 2$ bits per symbol period (bpsp). We note that $k_{1}+k_{2}$ is divided by two, because the transmitted bitstream requires two symbol periods to reach the DN.

A special case of the above transmission mechanism, called Dual-Hop Hybrid Space Shift Keying (DH-HSSK), is formed if we set $s_{k}=1$. In such a scenario, the information is conveyed using only the index $i$ of the receiving $\mathrm{RN}$.

\section{Linear PRECoding at the SN USing Practical SCENARIOS OF I-CSIT}

When P-CSIT is available at the SN, the design of the precoding matrix $\mathbf{P}$ is trivial, as in Section II. Our aim in the next two subsection is to design the precoding matrix of (1) using I-CSIT.

\section{A. Imperfect Channel Knowledge at the Transmitter}

In practice, CSI is acquired by the receiver using a pilot sequence. Under the assumption that the channel does not change during one block of symbols, Channel State Information at the Receiver (CSIR) may be considered accurate. When the channel is rapidly varying, even CSIR is subjected to imperfections. At the transmitter side, CSI is acquired either using a low rate feedback from the receiver or using the reciprocity principle where is applicable (pure TDD). In the general case, channel imperfections at the transmitter side may occur because of: i) the channel estimation errors; ii) the quantization errors when a reverse feedback link is employed; and iii) the outdated version of CSIT (time varying channel).

Clearly, in practical scenarios the acquisition of perfect (accurate) CSI is either a very expensive or even an unrealistic process. Hence, there is a high need for designs that take into account channel imperfections. A widely used channel model, that captures channel imperfections, expresses the channel matrix as [37],

$$
\mathbf{H}_{\mathrm{SR}}=\overline{\mathbf{H}}_{\mathrm{SR}}+\widetilde{\mathbf{H}}_{\mathrm{SR}}
$$

In (10), $\overline{\mathbf{H}}_{\mathrm{SR}}$ represents the long term channel evolution which can be accurately acquired by the transmitter. Moreover, $\widetilde{\mathbf{H}}_{\mathrm{SR}}$ denotes the channel for which only some kind of statistical or worst-case knowledge is considered possible. A common assumption is to model the long term channel evolution as $\overline{\mathbf{H}}_{\mathrm{SR}} \sim \mathcal{C} \mathcal{N}\left(\mathbf{0}, \sigma_{\overline{\mathbf{H}}_{\mathrm{SR}}}^{2} \mathbf{I}\right)$, while $\widetilde{\mathbf{H}}_{\mathrm{SR}} \sim \mathcal{C N}\left(\mathbf{0}, \sigma_{\widetilde{\mathbf{H}}_{\mathrm{SR}}}^{2} \mathbf{I}\right)$. We note that each element of $\overline{\mathbf{H}}_{\mathrm{SR}}$ remains constant over a block of symbols. However, the elements of $\widetilde{\mathbf{H}}_{\mathrm{SR}}$ change every symbol period. $\mathbf{H}_{\mathrm{SR}}$ denotes the instantaneous channel matrix. Finally, in order to avoid any power amplification due to the wireless channel, we assume $\sigma_{\overline{\mathbf{H}}_{\mathrm{SR}}}^{2}+\sigma_{\widetilde{\mathbf{H}}_{\mathrm{SR}}}^{2}=1$.

In the following two subsections, we design the precoding matrix $\mathbf{P}$ at the $\mathrm{SN}$ using two methods from optimization theory, Stochastic Robust Approximation (SRA) and Worst-Case Robust Approximation (WCRA) [38]. SRA follows a statistical approach and solves the resulting minimization problems over their expectation. In contrast, WCRA is a worst-case method and solves the same minimization problems assuming that $\left\|\widetilde{\mathbf{H}}_{\mathrm{SR}}\right\|_{2} \leq \alpha$. We note that both approaches employ I-CSIT in a statistical or scalar form which does not change rapidly over time.

\section{B. Objective Function}

In this subsection, we present the objective function that we minimize in order to design the precoding matrix $\mathbf{P}$ using I-CSIT. When P-CSIT is available, the ZF precoding matrix of (1) is just the pseudo-inverse matrix of $\mathbf{H}_{\mathrm{SR}}$. An alternative 
way to obtain the ZF precoder is to solve the following $N_{R}$ minimization problems:

$$
\min _{\mathbf{p}_{l}}\left\|\mathbf{H}_{\mathrm{SR}} \mathbf{p}_{l}-\mathbf{e}_{l}\right\|_{2}^{2}, \quad \forall l=1, \ldots, N_{R},
$$

where $\mathbf{e}_{l}$ is the $l$-th column of the identity matrix $\mathbf{I}_{N_{R}, N_{R}}=$ $\left[\mathbf{e}_{1}, \ldots, \mathbf{e}_{N_{R}}\right]$.

\section{ZF-Like Precoding Based on SRA}

When SRA is employed, the minimization problems of (11) are solved over their expectation [38]

$$
\min _{\mathbf{p}_{l}} \mathrm{E}\left[\left\|\mathbf{H}_{\mathrm{SR}} \mathbf{p}_{l}-\mathbf{e}_{l}\right\|_{2}^{2}\right], \quad \forall l=1, \ldots, N_{R} .
$$

If we use the fact that the MIMO channel can be written as in (10), where $\mathrm{E}\left[\widetilde{\mathbf{H}}_{\mathrm{SR}}\right]=\mathbf{0}$, (12) can be reformulated as:

$$
\min _{\mathbf{p}_{l}}\left\{\left\|\overline{\mathbf{H}}_{\mathrm{SR}} \mathbf{p}_{l}-\mathbf{e}_{l}\right\|_{2}^{2}+\mathbf{p}_{l}^{H} \mathrm{E}\left[\widetilde{\mathbf{H}}_{\mathrm{SR}}^{H} \widetilde{\mathbf{H}}_{\mathrm{SR}}\right] \mathbf{p}_{l}\right\},
$$

which is obviously a convex optimization problem (sum of quadratic functions). Thus, the solution can be achieved by setting its gradient equal to zero. In this case, the analytical solution is written as:

$$
\mathbf{p}_{l}=\left[\overline{\mathbf{H}}_{\mathrm{SR}}^{H} \overline{\mathbf{H}}_{\mathrm{SR}}+\mathrm{E}\left[\widetilde{\mathbf{H}}_{\mathrm{SR}}^{H} \widetilde{\mathbf{H}}_{\mathrm{SR}}\right]\right]^{-1} \overline{\mathbf{H}}_{\mathrm{SR}}^{H} \mathbf{e}_{l},
$$

or

$$
\mathbf{P}_{\mathrm{SRA}}=\left[\overline{\mathbf{H}}_{\mathrm{SR}}^{H} \overline{\mathbf{H}}_{\mathrm{SR}}+\mathrm{E}\left[\widetilde{\mathbf{H}}_{\mathrm{SR}}^{H} \widetilde{\mathbf{H}}_{\mathrm{SR}}\right]\right]^{-1} \overline{\mathbf{H}}_{\mathrm{SR}}^{H}
$$

in a collective matrix form. A careful look in $\mathrm{E}\left[\widetilde{\mathbf{H}}_{\mathrm{SR}}^{H} \widetilde{\mathbf{H}}_{\mathrm{SR}}\right]$ reveals its structured form. For example, when $\widetilde{\mathbf{H}}_{\mathrm{SR}}$ is uncorrelated, we have:

$$
\mathrm{E}\left[\widetilde{\mathbf{H}}_{\mathrm{SR}}^{H} \widetilde{\mathbf{H}}_{\mathrm{SR}}\right]=N_{R} \sigma_{\widetilde{\mathbf{H}}_{\mathrm{SR}}}^{2} \mathbf{I}_{N_{t}, N_{t}} .
$$

Thus, the computation of the previous quantity requires only the knowledge of $\sigma_{\widetilde{\mathbf{H}}_{\mathrm{SR}}}^{2}$, which is scalar.

\section{ZF-Like Precoding Based on WCRA}

When WCRA is utilized, the MIMO wireless channel is expressed as the non-empty and bounded set $\Phi \subseteq \mathcal{C}^{N_{R}, N_{t}}$. Here, $\Phi$ represents all the possible values of the channel matrix $\mathbf{H}_{\mathrm{SR}}$. Given a feasible precoding vector $\mathbf{p}_{l}$, the worst case error can be formulated as $e_{\mathrm{wc}}\left(\mathbf{p}_{l}\right)=\sup \left[\left\|\mathbf{H}_{\mathrm{SR}} \mathbf{p}_{l}-\mathbf{e}_{l}\right\|_{2} \mid \mathbf{H} \in \Phi\right]$. Our aim in this subsection is to design a precoder that minimizes the worst case error $e_{\mathrm{wc}}\left(\mathbf{p}_{l}\right)$. Under this aim, the minimization problem can be formulated as:

$$
\min _{\mathbf{p}_{l}} \sup \left[\left\|\mathbf{H}_{\mathrm{SR}} \mathbf{p}_{l}-\mathbf{e}_{l}\right\|_{2} \mid \mathbf{H}_{\mathrm{SR}} \in \Phi\right] \quad \forall l=1, \ldots, N_{R} .
$$

In this paper, we employ the Norm Bound Error (NBE) method from [38]. In NBE, the uncertainty of $\widetilde{\mathbf{H}}_{\mathrm{SR}}$ is considered within a norm ball of radius $\alpha$. Thus, the set $\Phi$ is written as:

$$
\Phi=\left\{\mathbf{H}_{\mathrm{SR}}=\overline{\mathbf{H}}_{\mathrm{SR}}+\widetilde{\mathbf{H}}_{\mathrm{SR}}\left\|\widetilde{\mathbf{H}}_{\mathrm{SR}}\right\|_{2} \leq \alpha\right\},
$$

where $\alpha>0$. Let

$$
e_{\mathrm{wc}}^{\mathrm{NBE}}\left(\mathbf{p}_{l}\right)=\sup \left\{\left\|\overline{\mathbf{H}}_{\mathrm{SR}} \mathbf{p}_{l}-\mathbf{e}_{l}+\widetilde{\mathbf{H}}_{\mathrm{SR}} \mathbf{p}_{l}\right\|_{2} \mid\left\|\widetilde{\mathbf{H}}_{\mathrm{SR}}\right\|_{2} \leq \alpha\right\}
$$

be the worst-case error given the precoding vector $\mathbf{p}_{l}$. After some arithmetic manipulations, it is shown that $e_{\mathrm{wc}}^{\mathrm{NBE}}\left(\mathbf{p}_{l}\right)$ is equal to

$$
e_{\mathrm{wc}}^{\mathrm{NBE}}\left(\mathbf{p}_{l}\right)=\left\|\overline{\mathbf{H}}_{\mathrm{SR}} \mathbf{p}_{l}-\mathbf{e}_{l}\right\|_{2}+\alpha\left\|\mathbf{p}_{l}\right\|_{2}
$$

and it is attained for $\widetilde{\mathbf{H}}_{\mathrm{SR}}=\alpha \mathbf{u v}{ }^{H}$, where

$$
\mathbf{u}=\frac{\overline{\mathbf{H}}_{\mathrm{SR}} \mathbf{p}_{l}-\mathbf{e}_{l}}{\left\|\overline{\mathbf{H}}_{\mathrm{SR}} \mathbf{p}_{l}-\mathbf{e}_{l}\right\|_{2}} \text { and } \mathbf{v}=\frac{\mathbf{p}_{l}}{\left\|\mathbf{p}_{l}\right\|_{2}},
$$

given that

$$
\overline{\mathbf{H}}_{\mathrm{SR}} \mathbf{p}_{l}-\mathbf{e}_{l} \neq \mathbf{0} \text { and } \mathbf{p}_{l} \neq \mathbf{0} .
$$

Thus, the minimization problem of (17) can be reformulated as:

$$
\min _{\mathbf{p}_{l}}\left\|\overline{\mathbf{H}}_{\mathrm{SR}} \mathbf{p}_{l}-\mathbf{e}_{l}\right\|_{2}+\alpha\left\|\mathbf{p}_{l}\right\|_{2} .
$$

The minimization problems of (18) can be transformed to:

$$
\begin{aligned}
& \min _{\left\{t_{1}, t_{2}\right\}} t_{1}+a t_{2} \\
& \text { subject to }\left\|\overline{\mathbf{H}}_{\mathrm{SR}} \mathbf{p}_{l}-\mathbf{e}_{l}\right\|_{2} \leq t_{1},\left\|\mathbf{p}_{l}\right\|_{2} \leq t_{2},
\end{aligned}
$$

which is solved as a Second Order Cone Programming (SOCP) problem using the interior point method [38]. Alternatively, (18) can be transformed in a TR form as:

$$
\min _{\mathbf{p}_{l}}\left\|\overline{\mathbf{H}}_{\mathrm{SR}} \mathbf{p}_{l}-\mathbf{e}_{l}\right\|_{2}^{2}+\beta\left\|\mathbf{p}_{l}\right\|_{2}^{2},
$$

for some value of $\beta$ [38].

Again, (20) is convex because $e_{\mathrm{wc}}^{\mathrm{NBE}}\left(\mathbf{p}_{l}\right)$ is the sum of quadratic functions. Thus, the solution can be reached using the gradient condition and is written as:

$$
\mathbf{p}_{l}=\left[\overline{\mathbf{H}}_{\mathrm{SR}}^{H} \overline{\mathbf{H}}_{\mathrm{SR}}+\beta \mathbf{I}\right]^{-1} \overline{\mathbf{H}}_{\mathrm{SR}}^{H} \mathbf{e}_{l} .
$$

The collective matrix form of the WCRA precoder is expressed as:

$$
\mathbf{P}_{\mathrm{WCRA}}=\left[\overline{\mathbf{H}}_{\mathrm{SR}}^{H} \overline{\mathbf{H}}_{\mathrm{SR}}+\beta \mathbf{I}\right]^{-1} \overline{\mathbf{H}}_{\mathrm{SR}}^{H} .
$$

\section{E. Precoding in the Presence of Transmit and Receive Space Correlations}

The problems of (13) and (20) are different forms of Tikhonov Regularization (TR) [38]. A valuable property of TR theory is that it does not pose any rank restriction on the involved matrices $\overline{\mathbf{H}}_{\mathrm{SR}}$ and $\widetilde{\mathbf{H}}_{\mathrm{SR}}$ as long as the matrices $\overline{\mathbf{H}}_{\mathrm{SR}}^{H} \overline{\mathbf{H}}_{\mathrm{SR}}+\mathrm{E}\left[\widetilde{\mathbf{H}}_{\mathrm{SR}}^{H} \widetilde{\mathbf{H}}_{\mathrm{SR}}\right]$ and $\overline{\mathbf{H}}_{\mathrm{SR}}^{H} \overline{\mathbf{H}}_{\mathrm{SR}}+\beta \mathbf{I}$ are positive 
definite [38]. Thus, our analytical solutions of (15) and (22) may enjoy the additional merit of being applicable to spatially correlated channels.

In this paper, we employ the Kronecker correlation model [39]. According to this correlation model, the MIMO channel can be rewritten as:

$$
\mathbf{H}_{\mathrm{SR}}=\mathbf{R}_{R}^{1 / 2} \mathbf{H}_{w}\left(\mathbf{R}_{T}^{1 / 2}\right)^{T}
$$

where $\mathbf{H}_{w} \sim \mathcal{C N}(\mathbf{0}, \mathbf{I}) . \mathbf{R}_{T}$ and $\mathbf{R}_{R}$ represent the transmit and receive spatial correlation matrices, respectively. In this case the wireless channel is distributed as $\mathbf{H}_{\mathrm{SR}} \sim \mathcal{C N}\left(\mathbf{0}, \mathbf{R}_{T} \otimes \mathbf{R}_{R}\right)$. Usually, the entries of the spatial correlation matrices $\mathbf{R}_{R}$ and $\mathbf{R}_{T}$ are generated using an exponential model with $R_{T}(i, j)=$ $\rho_{t}^{|i-j|}$ and $R_{R}(i, j)=\rho_{r}^{|i-j|}$, where $0 \leq \rho_{t}, \rho_{r} \leq 1$. Values of $\rho_{t}$ and $\rho_{r}$ close to 0 mean low correlation, whereas values close to 1 mean high correlations.

If we combine the Kronecker correlation model of (23) with the model of I-CSIT described in Section III-A, the MIMO channel is expressed as:

$$
\mathbf{H}_{\mathrm{SR}}=\mathbf{R}_{R}^{1 / 2}\left(\overline{\mathbf{H}}_{\mathrm{SR}}+\widetilde{\mathbf{H}}_{\mathrm{SR}}\right)\left(\mathbf{R}_{T}^{1 / 2}\right)^{T} .
$$

Here, the matrix $\mathbf{R}_{R}^{1 / 2} \overline{\mathbf{H}}_{\mathrm{SR}}\left(\mathbf{R}_{T}^{1 / 2}\right)^{T}$ represents the fully known part of the channel and the matrix $\mathbf{R}_{R}^{1 / 2} \widetilde{\mathbf{H}}_{\mathrm{SR}}\left(\mathbf{R}_{T}^{1 / 2}\right)^{T}$ represents the channel uncertainty.

The inspection of (15) and (22) reveals that only the analytical form of the design based on SRA is affected by the correlated channel. This is because SRA requires the computation of $\mathrm{E}\left[\widetilde{\mathbf{H}}_{\mathrm{SR}}^{H} \widetilde{\mathbf{H}}_{\mathrm{SR}}\right]$. After some arithmetic manipulations this quantity is expressed as:

$$
\mathrm{E}\left[\widetilde{\mathbf{H}}_{\mathrm{SR}}^{H} \widetilde{\mathbf{H}}_{\mathrm{SR}}\right]=\sigma_{\widetilde{\mathbf{H}}_{\mathrm{SR}}^{2}} \operatorname{tr}\left(\mathbf{R}_{R}\right) \mathbf{R}_{T}^{1 / 2}\left(\mathbf{R}_{T}^{1 / 2}\right)^{T} .
$$

\section{F. Instantaneous Transmit Power Analysis}

In this subsection, we focus on the $\mathrm{SN}$ and study its transmission power. Given that $\mathrm{E}_{\mathbf{x}}\left[\|\mathbf{x}\|_{2}^{2}\right]=1$, the instantaneous transmission power $\|\mathbf{P D x}\|_{2}^{2}$ is fully characterized by the quantity $P_{\mathrm{S}}=\|\mathbf{P D}\|_{2}^{2}$.

In (1), we employ the normalization matrix $\mathbf{D}$ in order to make sure that the transmission power at the $\mathrm{SN}$ is not amplified by the precoder $\mathbf{P}$ in adverse channel conditions. As shown in the following, the proposed precoders have a structure that imposes an indirect transmit power constraint. The practical value of this constraint is that we are able to set $\mathbf{D}=\mathbf{I}_{N_{R}, N_{R}}$, without amplifying the power transmission to an unacceptable level. Additionally, we can assume that $\mathbf{H}_{\mathrm{SR}} \mathbf{P} \approx \mathbf{I}_{N_{R}, N_{R}}$ (when $\mathbf{P}$ is the MMSE, or SRA, or WCRA precoder) in order to keep the complexity of the RNs low. In this way, there is no need for $d_{i}$ to be known at the RNs. Hence, the detection algorithm of (7) is further simplified by setting $d_{i}=1$. Though, we emphasize that in the BER simulation results of Section VI the precoding designs of SRA and WCRA are normalized as described in Section II. We follow this choice for the sake of fair comparison with the other precoding methods (same transmission power).
SRA and WCRA are forms of TR when I-CSIT is available. As a consequence, we expect both designs to offer a reduced transmit power [38]. Let us begin our proof by taking the SVD of $\overline{\mathbf{H}}_{\mathrm{SR}}$ :

$$
\overline{\mathbf{H}}_{\mathrm{SR}}=\overline{\mathbf{U}} \overline{\mathbf{\Sigma}} \overline{\mathbf{V}}^{H} .
$$

Here, $\overline{\mathbf{U}}$ and $\overline{\mathbf{V}}$ are unitary matrices and $\overline{\boldsymbol{\Sigma}}$ is a diagonal matrix containing at its main diagonal the $N_{R}$ singular values $\bar{\sigma}_{i}$ of $\overline{\mathbf{H}}_{\mathrm{SR}}$ (without loss of generality we assume that $\overline{\mathbf{H}}_{\mathrm{SR}}$ has a rank of $N_{R}$ ). If we plug (26) into (15) and after some arithmetic manipulations, the SRA precoder is written as:

$$
\mathbf{P}_{\mathrm{SRA}}=\overline{\mathbf{V}}\left[\overline{\mathbf{D}}+N_{R} \sigma_{\widetilde{\mathbf{H}}_{\mathrm{SR}}}^{2} \mathbf{I}_{N_{t}, N_{t}}\right]^{-1} \overline{\mathbf{\Sigma}}^{T} \overline{\mathbf{U}}^{H},
$$

where $\overline{\mathbf{D}}$ is a $N_{t} \times N_{t}$ diagonal matrix equal to $\overline{\mathbf{D}}=$ $\operatorname{diag}\left(\bar{\sigma}_{1}^{2}, \ldots, \bar{\sigma}_{N_{R}}^{2}\right) \cdot \bar{\sigma}_{i}^{2}, i=1, \ldots, N_{R}$ are the eigenvalues of $\overline{\mathbf{H}}_{\mathrm{SR}}$.

Taking into account that both $\overline{\mathbf{D}}+N_{R} \sigma_{\widetilde{\mathbf{H}}_{\mathrm{SR}}}^{2} \mathbf{I}_{N_{t}, N_{t}}$ and $\overline{\boldsymbol{\Sigma}}$ are diagonal matrices, we can further proceed with (27) and show that

$$
\mathbf{P}_{\mathrm{SRA}}=\overline{\mathbf{V}} \overline{\mathbf{\Sigma}}_{\mathrm{SRA}} \overline{\mathbf{U}}^{H}
$$

where

$$
\Sigma_{\mathrm{SRA}}=\operatorname{diag}\left(\frac{\bar{\sigma}_{1}}{\bar{\sigma}_{1}^{2}+N_{R} \sigma_{\widetilde{\mathbf{H}}_{\mathrm{SR}}}^{2}}, \ldots, \frac{\bar{\sigma}_{N_{R}}}{\bar{\sigma}_{N_{R}}^{2}+N_{R} \sigma_{\widetilde{\mathbf{H}}_{\mathrm{SR}}}^{2}}\right) .
$$

In order to evaluate the transmit power of the SRA precoder, we have to compute the Frobenius norm of the precoding matrix of (28)

$$
\left\|\mathbf{P}_{\mathrm{SRA}}\right\|_{F}^{2}=\operatorname{tr}\left(\mathbf{P}_{\mathrm{SRA}}^{H} \mathbf{P}_{\mathrm{SRA}}\right)=\operatorname{tr}\left(\overline{\mathbf{U}} \boldsymbol{\Sigma}_{\mathrm{SRA}}^{T} \boldsymbol{\Sigma}_{\mathrm{SRA}} \overline{\mathbf{U}}^{H}\right) .
$$

Using the argument that the trace operator is invariant under cyclic permutations $\operatorname{tr}(\mathbf{A B C})=\operatorname{tr}(\mathbf{C A B})$, we can show that

$$
\left\|\mathbf{P}_{\mathrm{SRA}}\right\|_{F}^{2}=\sum_{i=1}^{N_{R}}\left(\frac{\bar{\sigma}_{i}}{\bar{\sigma}_{i}^{2}+N_{R} \sigma_{\widetilde{\mathbf{H}}_{\mathrm{SR}}}^{2}}\right)^{2} .
$$

In order to quantify the maximum of $\left\|\mathbf{P}_{\mathrm{SRA}}\right\|_{F}^{2}$, we focus on each component

$$
\theta_{i}=\frac{\bar{\sigma}_{i}}{\bar{\sigma}_{i}^{2}+N_{R} \sigma_{\widetilde{\mathbf{H}}_{\mathrm{SR}}}^{2}}
$$

of the summation of (31). Given that $\bar{\sigma}_{i}$ and $\sigma_{\widetilde{\mathbf{H}}_{\mathrm{SR}}}^{2}$ take positive values, it is clear to see that $\theta_{i}$ : i) is close to zero when $\bar{\sigma}_{i} \rightarrow$ 0 ; ii) it attains the maximum value of $1 /\left(1+N_{R} \sigma_{\widetilde{\mathbf{H}}_{\mathrm{SR}}}^{2}\right)<1$ when $\bar{\sigma}_{i}=N_{R} \sigma_{\widetilde{\mathbf{H}}_{\mathrm{SR}}}^{2}$; and iii) approaches zero when $\bar{\sigma}_{i} \rightarrow+\infty$. Hence, the instantaneous transmit power of $\mathbf{P}_{\mathrm{SRA}}$ is always constrained by $\left\|\mathbf{P}_{\mathrm{SRA}}\right\|_{F}^{2}<N_{R}$, since $\bar{\sigma}_{i} /\left(\bar{\sigma}_{i}^{2}+N_{R} \sigma_{\widetilde{\mathbf{H}}_{\mathrm{SR}}}^{2}\right)<1$ for every positive value of $\bar{\sigma}_{i}$ and $\sigma_{\widetilde{\mathbf{H}}_{\mathrm{SR}}}^{2}$.

Again, following the same steps, we can express the WCRA precoder as:

$$
\mathbf{P}_{\mathrm{WCRA}}=\overline{\mathbf{V}} \overline{\mathbf{\Sigma}}_{\mathrm{WCRA}} \overline{\mathbf{U}}^{H},
$$


where

$$
\boldsymbol{\Sigma}_{\mathrm{WCRA}}=\operatorname{diag}\left(\frac{\bar{\sigma}_{1}}{\bar{\sigma}_{1}^{2}+\beta}, \ldots, \frac{\bar{\sigma}_{N_{R}}}{\bar{\sigma}_{N_{R}}^{2}+\beta}\right) .
$$

The transmit power of WCRA is expressed as:

$$
\left\|\mathbf{P}_{\mathrm{WCRA}}\right\|_{F}^{2}=\sum_{i=1}^{N_{R}}\left(\frac{\bar{\sigma}_{i}}{\bar{\sigma}_{i}^{2}+\beta}\right)^{2}<N_{R} .
$$

Clearly, $\left\|\mathbf{P}_{\text {WCRA }}\right\|_{F}^{2}$ is also constrained because $\left(\bar{\sigma}_{i} / \bar{\sigma}_{i}^{2}+\right.$ $\beta)<1$ for every $\left\{\bar{\sigma}_{i}, \beta\right\}>0$.

A careful inspection of (31) and (35) reveals that the channel uncertainty (in the form of $\sigma_{\widetilde{\mathbf{H}}_{\mathrm{SR}}}^{2}$ for SRA and in the form of $\beta$ for WCRA) acts as a regularization parameter which adjusts the transmitted power. When the channel uncertainty approaches to zero, both SRA and WCRA reduce to ZF precoding and the transmitted power is maximized up to the transmitted power of ZF precoding. In contrast, when the channel uncertainty is increased, the level of power transmission imposed by the transmitter is decreased. This means that when the channel knowledge at the transmitter becomes more inaccurate, the transmitter reduces the transmitted power in order to avoid further degradation.

In the next few lines, we express without proof (due to space limitation) the transmit power behavior of ZF and MMSE precoding when I-CSIT is available at the SN. Using a similar approach as previously, it is shown that the transmit power of ZF precoding is:

$$
\left\|\mathbf{P}_{\mathrm{ZF}}\right\|_{F}^{2}=\sum_{i=1}^{N_{R}} \frac{1}{\bar{\sigma}_{i}}
$$

and the transmit power of MMSE precoding is:

$$
\left\|\mathbf{P}_{\mathrm{MMSE}}\right\|_{F}^{2}=\sum_{i=1}^{N_{R}}\left(\frac{\bar{\sigma}_{i}}{{\overline{\sigma_{i}}}^{2}+\mu}\right)^{2} .
$$

From (36), it is clear that the power transmission of ZF precoding is increased to unacceptable high levels when the channel is in deep fade $\left(\bar{\sigma}_{i}^{2} \rightarrow 0\right)$. In contrast, for MMSE precoding it can be shown that the power transmission is constrained by $\left\|\mathbf{P}_{\text {MMSE }}\right\|_{F}^{2}<N_{R}$ because $\bar{\sigma}_{i} /\left(\bar{\sigma}_{i}^{2}+\mu\right)<1, i=1, \ldots, N_{R}$. A similar proof for the ZF and MMSE precoders is attained when the SN employs P-CSIT. In this case, the power transmission of the ZF and MMSE is given from (36) and (37) by replacing $\bar{\sigma}_{i}$ with $\sigma_{i}$, where the eigenvalues of $\mathbf{H}_{\mathrm{SR}}$ are denoted as $\sigma_{i}^{2}$.

Finally, the inspection of (31), (35), and (37) shows that there is a relation between the transmission power of different precoding methods. It is easy to demonstrate, using the structure of the power transmission of each precoder, that MMSE imposes higher instantaneous power transmission than: i) SRA when $\sigma_{\widetilde{\mathbf{H}}_{\mathrm{SR}}}^{2}>\mu / N_{R}$, and ii) WCRA when $\beta>\mu$.

\section{G. Average Transmit Power Analysis}

In this subsection, we provide the analytical derivation of the average precoding power $\bar{P}_{\mathrm{s}}$ of the methods employed in this work when: i) I-CSIT is available and ii) $\mathbf{D}=\mathbf{I}_{N_{R}, N_{R}}$. The inspection of (31), (35)-(37) reveals that the instantaneous power of each precoder has the following structured form:

$$
\|\mathbf{P}\|_{F}^{2}=\sum_{i=1}^{N_{R}}\left(\frac{\bar{\sigma}_{i}}{{\overline{\sigma_{i}}}^{2}+\vartheta}\right)^{2}=\sum_{i=1}^{N_{R}}\left(\frac{\sqrt{\bar{\lambda}_{i}}}{\bar{\lambda}_{i}+\vartheta}\right)^{2} .
$$

where, depending on the precoding method, $\vartheta$ takes the following values: i) $\vartheta=0$ for ZF; ii) $\vartheta=\mu$ for MMSE; iii) $\vartheta=N_{R} \sigma_{\widetilde{\mathbf{H}}_{\mathrm{SR}}}^{2}$ for SRA; and iv) $\vartheta=\beta$ for WCRA. In (38), for notational convenience, we substitute ${\overline{\sigma_{i}}}^{2}$ with $\bar{\lambda}_{i}$.

The analytical derivation of the average power of all precoding methods is given as:

$$
\bar{P}_{s}=\mathrm{E}\left[\|\mathbf{P}\|_{F}^{2}\right]=\mathrm{E}_{\bar{\tau}}\left[\sum_{i=1}^{N_{R}}\left(\frac{\sqrt{\bar{\lambda}_{i}}}{\bar{\lambda}_{i}+\vartheta}\right)^{2}\right],
$$

where $\overline{\boldsymbol{\tau}}=\left[\bar{\lambda}_{1}, \ldots, \bar{\lambda}_{N_{R}}\right]^{T}$. It is not difficult to see that the random vector $\bar{\tau}$ contains the unordered eigenvalues of the Wishart matrix $\overline{\mathbf{W}}=\overline{\mathbf{H}}_{\mathrm{SR}} \overline{\mathbf{H}}_{\mathrm{SR}}^{H} \sim \mathcal{C W}\left(N_{R}, \boldsymbol{\Sigma}_{\overline{\mathbf{H}}_{\mathrm{SR}}}\right)$, where $\mathcal{C W}\left(N_{R}, \boldsymbol{\Sigma}_{\overline{\mathbf{H}}_{\mathrm{SR}}}\right)$ denotes a central complex Wishart distribution as defined in [40, eq. 2.6]. $\Sigma_{\overline{\mathbf{H}}_{\mathrm{SR}}}$ is the covariance matrix of $\overline{\mathbf{H}}_{\mathrm{SR}}$. In the following, we derive the expectation of (39) when the $\mathrm{SN}-\mathrm{RNs}$ channel is : i) uncorrelated and ii) transmit correlated $\left(\mathbf{R}_{R}=\mathbf{I}_{N_{R}, N_{R}}\right)$. The latter case corresponds to the scenario where the transmit antennas belongs to a single transmitter, while the receive antennas belong to RNs placed sufficiently apart.

1) Uncorrelated $S N-R N s$ Channel: When the $\mathrm{SN}-\mathrm{RNs}$ is uncorrelated and distributed as described in Section III-A, the covariance matrix of $\overline{\mathbf{H}}_{\mathrm{SR}}$ equals to $\boldsymbol{\Sigma}_{\overline{\mathbf{H}}_{\mathrm{SR}}}=\sigma_{\mathbf{H}_{\mathrm{SR}}}^{2}$ I. Given that $\overline{\mathbf{H}}_{\mathrm{SR}}=\sqrt{\sigma_{\overline{\mathbf{H}}_{\mathrm{SR}}}^{2}} \mathbf{H}_{w}$, where $\mathbf{H}_{w} \sim \mathcal{C N}(\mathbf{0}, \mathbf{I})$, the eigenvalues $\bar{\lambda}_{i}$ of $\overline{\mathbf{H}}_{\mathrm{SR}}$ can be rewritten as $\bar{\lambda}_{i}=\sqrt{\sigma_{\mathbf{H}_{\mathrm{SR}}^{2}}^{2}} \lambda_{i}$. Here, $\lambda_{i}$, $i=1, \ldots, N_{R}$, represent the eigenvalues of $\mathbf{H}_{w}$.

If we take into account the previous relation and set : i) $\bar{\tau}=$ $\sqrt{\sigma_{\mathbf{H}_{\mathrm{SR}}}^{2}} \tau$ and ii) $\phi=\vartheta / \sqrt{\sigma_{\mathbf{H}_{\mathrm{SR}}}^{2}}$, after some manipulations, (39) is transformed to

$$
\bar{P}_{s}=\frac{1}{\sigma_{\mathbf{H}_{\mathrm{SR}}}^{2}} \mathrm{E}_{\boldsymbol{\tau}}\left[\sum_{i=1}^{N_{R}} \frac{\lambda_{i}}{\left(\lambda_{i}+\phi\right)^{2}}\right]=\frac{N_{R}}{\sigma_{\overline{\mathbf{H}}_{\mathrm{SR}}}^{2}} \mathrm{E}_{\lambda}\left[\frac{\lambda}{(\lambda+\phi)^{2}}\right] .
$$

In (40), $\lambda$ denotes the unordered eigenvalues of the complex central Wishart matrix $\mathbf{W}=\mathbf{H}_{w} \mathbf{H}_{w}^{H}$. The marginal probability density function (p.d.f.) of $\lambda$ is given in [40, eq. 2.23] as:

$$
f_{\lambda}(x)=\frac{1}{N_{R}}\left[\sum_{k=0}^{N_{R}-1} \frac{k !}{(k+\Delta) !}\left[L_{k}^{\Delta}(x)\right]^{2} x^{\Delta} e^{-x}\right] H_{0}(x),
$$

where $\Delta=N_{t}-N_{R}$, and

$$
L_{k}^{\Delta}(x)=\sum_{i=0}^{k}(-1)^{i}\left(\begin{array}{c}
k+\Delta \\
k-i
\end{array}\right) \frac{x^{i}}{i !} .
$$


Here, $L_{k}^{\Delta}(x)$ denotes the Laguerre polynomials of degree $k$. In addition, $H_{0}(x)$ is the Heaviside step function, for which it holds that $H_{0}(x)=0$ for $x<0$ and $H_{0}(x)=1$ for $x \geq 0$.

Thus, if we evaluate the expectation

$$
\mathrm{E}_{\lambda}\left[\frac{\lambda}{(\lambda+\phi)^{2}}\right]=\int_{0}^{+\infty} \frac{x}{(x+\phi)^{2}} f_{\lambda}(x) d x
$$

using (41) (in a lengthy procedure which is omitted due to space limitation), the average precoding power of (40) becomes:

$$
\begin{aligned}
\bar{P}_{s}= & \frac{1}{\sigma_{\mathbf{H}_{\mathrm{SR}}}^{2}} \sum_{k=0}^{N_{R}-1} \frac{k !}{(k+\Delta) !} \sum_{l=0}^{k} \sum_{m=0}^{k} \frac{(-1)^{l+m}}{l ! m !}\left(\begin{array}{c}
k+\Delta \\
k-l
\end{array}\right) \\
& \times\left(\begin{array}{c}
k+\Delta \\
k-m
\end{array}\right)(n+1) ! \phi^{n} e^{\phi}[\Gamma(-n, \phi)-\phi \Gamma(-n-1, \phi)] .
\end{aligned}
$$

In (44), $n=l+m+\Delta$ and $\Gamma(\cdot, \cdot)$ denotes the incomplete gamma function defined in [41, p. 899].

2) Transmit Correlated $S N-R N s$ Channel: The elaboration of (39) gives that:

$$
\bar{P}_{s}=\mathrm{E}_{\bar{\tau}}\left[\sum_{i=1}^{N_{R}}\left(\frac{\sqrt{\bar{\lambda}_{i}}}{\bar{\lambda}_{i}+\vartheta}\right)^{2}\right]=N_{R} \mathrm{E}_{\bar{\lambda}}\left[\frac{\bar{\lambda}}{\overline{(\lambda+\vartheta)^{2}}}\right],
$$

where $\bar{\lambda}$ is the unordered eigenvalues of the complex central Wishart matrix $\overline{\mathbf{W}}=\overline{\mathbf{H}}_{\mathrm{SR}} \overline{\mathbf{H}}_{\mathrm{SR}}^{H}$. Clearly, the evaluation of (45) requires the marginal p.d.f. of $\bar{\lambda}$, which is derived below.

The presence of only transmit space correlation in the wireless channel determines that $\overline{\mathbf{H}}_{\mathrm{SR}}=\mathbf{H}_{w}\left(\overline{\mathbf{R}}_{T}^{1 / 2}\right)^{T}$, where $\overline{\mathbf{R}}_{T}^{1 / 2}=\sqrt{\sigma_{\mathbf{H}_{\mathrm{SR}}}^{2}}\left(\mathbf{R}_{T}^{1 / 2}\right)^{T}$. Thus, $\overline{\mathbf{H}}_{\mathrm{SR}}$ is distributed according to the following complex Gaussian distribution $\mathbf{H}_{\mathrm{SR}} \sim$ $\mathcal{C N}\left(\mathbf{0}, \overline{\mathbf{R}}_{T} \otimes \mathbf{I}_{N_{R}, N_{R}}\right)$. In this case, the complex central Wishart matrix $\overline{\mathbf{W}}$ is distributed as $\mathcal{C} \mathcal{W}\left(N_{R}, \overline{\mathbf{R}}_{T}\right)$. For this reason, as it is shown in [42], the marginal p.d.f. of $\bar{\lambda}$ equals to:

$$
\begin{aligned}
f_{\bar{\lambda}}(x)=K & {\left[\sum _ { i = 1 } ^ { N _ { R } } \sum _ { j = 1 } ^ { N _ { R } } x ^ { j - 1 } \mathcal { D } ( i , j ) \left[\alpha_{\varrho+i}^{\varrho-1} e^{\frac{-x}{\alpha_{\varrho+i}}}\right.\right.} \\
& \left.\left.-\sum_{l=1}^{\varrho} \sum_{k=1}^{\varrho}\left[\boldsymbol{\Psi}^{-1}\right]_{k, l} \alpha_{\varrho+i}^{k-1} \alpha_{l}^{\varrho-1} e^{\frac{-x}{\alpha_{l}}}\right]\right] H_{0}(x),
\end{aligned}
$$

where $\varrho=N_{t}-N_{R}$ and

$$
K=\frac{\operatorname{det}(\mathbf{\Psi})}{N_{R}\left[\prod_{k<l}^{N_{t}}\left(\alpha_{l}-\alpha_{k}\right)\right] \prod_{l=1}^{N_{R}-1} l !} .
$$

$\Psi$ is the following $\varrho \times \varrho$ Vandermonde matrix

$$
\Psi=\left[\begin{array}{cccc}
1 & \alpha_{1} & \cdots & \alpha_{1}^{\varrho-1} \\
\vdots & \ddots & \ddots & \vdots \\
1 & \alpha_{\varrho} & \cdots & \alpha_{\varrho}^{\varrho-1}
\end{array}\right]
$$

In addition, $\alpha_{1}, \ldots, \alpha_{\varrho}$ represent the $\varrho$ distinct eigenvalues of $\overline{\mathbf{R}}_{T} \cdot \mathcal{D}(i, j)$ is the $(i, j)$ cofactor of the $N_{R} \times N_{R}$ matrix $\mathbf{C}$ whose $(l, k)$ element equals to

$$
[\mathbf{C}]_{l, k}=(k-1) !\left(\alpha_{\varrho+l}^{\varrho+k-1}-\sum_{p=1}^{\varrho} \sum_{q=1}^{\varrho}\left[\Psi^{-1}\right]_{p, q} \alpha_{\varrho+l}^{p-1} \alpha_{q}^{\varrho+k-1}\right) .
$$

At this point, given that the marginal p.d.f. of $\bar{\lambda}$ is available in (46), we are able to evaluate the following expectation

$$
\mathrm{E}_{\bar{\lambda}}\left[\frac{\bar{\lambda}}{\overline{(\lambda+\vartheta)^{2}}}\right]=\int_{0}^{+\infty} \frac{\bar{\lambda}}{(\bar{\lambda}+\vartheta)^{2}} f_{\bar{\lambda}}(x) d x
$$

and plug it in (45). Hence, we can prove that:

$$
\begin{aligned}
\bar{P}_{s}=K N_{R} \sum_{i=1}^{N_{R}} \sum_{j=1}^{N_{R}} \mathcal{D}(i, j)\left[\alpha_{\varrho+i}^{\varrho-1} g_{j}\left(\frac{1}{\alpha_{\varrho+i}}\right)\right. \\
\left.-\sum_{l=1}^{\varrho} \sum_{k=1}^{\varrho}\left[\Psi^{-1}\right]_{k, l} \alpha_{\varrho+i}^{k-1} \alpha_{l}^{\varrho-1} g_{j}\left(\frac{1}{\alpha_{l}}\right)\right],
\end{aligned}
$$

where

$$
g_{j}(y)=\vartheta^{j-1} e^{\vartheta y} j ![\Gamma(1-j, y \vartheta)-y \vartheta \Gamma(-j, y \vartheta)]
$$

and $\Gamma(\cdot)$ denotes the gamma function defined in [41, p. 892].

\section{Extension to a Distributed RELAYS ARCHITECTURE}

In some scenarios there is no backhaul-link between the RNs. This is the case when: i) the delay requirement does not allow communication between the RNs, ii) the cost of installing a backhaul connection is high, or iii) the RNs are remote terminals (like cell phones). This section extends the proposed centralized relay architecture from Section II to a distributed one with the employment of a decentralized detection algorithm at the RNs. All the other configurations of the proposed architecture remain the same.

During the first hop, the received signal at the $j$-th $\mathrm{RN}$ is described in (6). It is straightforward to see that if we set $s_{0}=$ 0 , (6) can be easily interpreted as the following $(M+1)$-ary Hypothesis Test (HT)

$$
H_{i}: y_{R j}=d_{j} s_{i}+w_{S R j}, \quad i=0, \ldots, M,
$$

which can be independently employed at each RN. In (50), $s_{i}$ takes values from $\left\{s_{0}, s_{1}, \ldots, s_{M}\right\}$. Clearly, the $H_{0}$ hypothesis $\left(s_{0}=0\right)$ corresponds to the case where the $j$-th $\mathrm{RN}$ is not the receiving node in the first hop. Thus, this RN remains silent during the second hop. On the other hand, all the other $H_{1}, \ldots, H_{M}$ hypotheses correspond to the case where the $j$ th node is the receiving/activating $\mathrm{RN}$ and the relayed conventional symbol is $s_{1}, \ldots, s_{M}$, respectively.

In this case, the distributed ML detector at each $\mathrm{RN}$ is reduced to the usual minimum distance rule

$$
\left(\hat{s}_{i}\right)=\arg \min _{s_{i} \in\left\{s_{0}, s_{1}, \ldots, s_{M}\right\}}\left|y_{R_{j}}-d_{j} s_{k}\right| .
$$


Given that $d_{j} s_{k}$ is precomputed and stored in the memory before the beginning of transmission, the computational complexity of (51) at each RN is just one complex subtractions per iteration.

Clearly, the independent execution of the detector of (51) at each RN could result into the activation of multiple RNs during the second hop. In the other extreme case, the distributed detectors of (51) of all RNs could detect the zero symbol $s_{0}$. When this extreme case occurs, all the RNs remain silent and no symbol is relayed to the DN. Due to the fact that the DN is unaware of this situation, its detector decides randomly based only on the ever present Gaussian noise.

In the literature, these problems are treated using error-aware detection at the DN [28], [32], which increases the complexity of the DN. In order to keep the complexity of the DN low, we decide to ignore the phenomenon of the activation of multiple RNs. Though, as it is demonstrated in Section VI, our DD algorithm benefits from the linear precoding at the SN, which results in high SNR gains at the RNs. Thus, the probability of activating multiple RNs is reduced. Because of this, practically and in most scenarios, either there is no performance difference from the CD algorithm, or there is a small BER penalty.

\section{Theoretical Evaluation of the Average Bit ERROR PROBABILITY}

In this section, we employ the well known union bound technique [34] in order to provide bounds on the ABEP of the proposed architecture. This is done both for the $\mathrm{CD}$ (Section II) and DD (Section IV) algorithms in the RNs. In order to provide tractability to our theoretical analysis, we focus on the scenario of ZF precoding with P-CSIT at the SN. We make this decision because it is well known that the ABEP performance analysis of MMSE or any other regularized ZF-like precoding method is a challenging problem [43]. Besides, in high SNRs, ZF precoding approaches the performance of MMSE precoding [36], [44].

\section{A. Average Bit Error Probability When the RNs Employ Centralized Detection}

When the CD algorithm of (7) is employed at the RNs, the DH-HSSK/HSM is a regenerative relay system with coordinated detection. Thus, we can follow a similar procedure such as [27], [45] and express the end-to-end ABEP as:

$$
P_{\mathrm{CD}}\left(\gamma_{1}, \gamma_{2}\right)=P_{1}\left(\gamma_{1}\right)+P_{2}\left(\gamma_{2}\right)-P_{1}\left(\gamma_{1}\right) P_{2}\left(\gamma_{2}\right) .
$$

In (52), $\gamma_{1}$ and $\gamma_{2}$ denotes the SNRs of the first and the second hop, respectively. $P_{\mathrm{CD}}\left(\gamma_{1}, \gamma_{2}\right)$ is the end-to-end ABEP, given $\gamma_{1}$ and $\gamma_{2}$. Furthermore, $P_{1}\left(\gamma_{1}\right)$ and $P_{2}\left(\gamma_{2}\right)$ represents the ABEP of the first and second hop, respectively. It is not difficult to see that $P_{1}\left(\gamma_{1}\right)$ and $P_{2}\left(\gamma_{2}\right)$ are the ABEP of R-SM and $\mathrm{SM}$, respectively. The ABEP of SM is widely studied in the literature [10], [11], [46], [47]. However, the ABEP of R-SM is a challenging problem, due to the use of ZF precoding [43].

We begin with the derivation of the ABEP of the first hop (ABEP of R-SM). We have to evaluate the Pairwise Error Probability (PEP) of transmitting $\mathrm{x}$ at the $\mathrm{RN}$ and receiving $\hat{\mathbf{x}}$ at the RNs. This is done for all possible combinations of $\mathbf{x}, \hat{\mathbf{x}} \in$ $\mathcal{A}_{1}=\left\{\mathbf{e}_{i} s_{k} \mid i \in\left\{1, \ldots, N_{R}\right\} \cap k \in\{1, \ldots, M\}\right\}$ and $\mathbf{x} \neq \hat{\mathbf{x}}$. Given that (5) holds and the ML detector selects the symbol $\mathbf{x}$ which minimizes the quantity $\left\|\mathbf{y}_{R}-\mathbf{D x}\right\|_{2}^{2}$, a symbol error occurs when $\mathcal{E}=\left\{\left\|\mathbf{y}_{R}-\mathbf{D x}\right\|_{2}^{2}>\left\|\mathbf{y}_{R}-\mathbf{D} \hat{\mathbf{x}}\right\|_{2}^{2}\right\}$. If we set $\mathbf{c}=\mathbf{x}-\hat{\mathbf{x}}$ and elaborate $\mathcal{E}$, it can be shown that $\mathcal{E}=$ $\left\{-\sum_{i=1}^{N_{R}} d_{i} \operatorname{Re}\left\{c_{i}^{*} \mathbf{w}_{S R_{i}}\right\}>\left(\mathbf{c}^{H} \mathbf{D c} / 2\right)\right\}$, where $c_{i}$ and $w_{S R_{i}}$ are the elements of $\mathbf{c}$ and $\mathbf{w}_{S R}$, respectively. If we consider that $-\sum_{i=1}^{N_{R}} d_{i} \operatorname{Re}\left\{c_{i}^{*} \mathbf{w}_{S R_{i}}\right\} \sim \mathcal{N}\left(0,\left(\sigma_{\mathbf{w}_{S R}}^{2} / 2\right) \mathbf{c}^{H} \mathbf{D}^{2} \mathbf{c}\right)$, it is shown that the instantaneous PEP conditioned on $\mathbf{D}^{2}$ is expressed as:

$$
P_{\mathrm{R}-\mathrm{SM}}\left(\mathbf{x} \rightarrow \hat{\mathbf{x}} \mid \mathbf{D}^{2}\right)=Q\left(\sqrt{\frac{\mathbf{c}^{H} \mathbf{D}^{2} \mathbf{c}}{2} \gamma_{1}}\right),
$$

where $\gamma_{1}=1 / \sigma_{\mathbf{w}_{S R}}^{2}$ and $Q(\cdot)$ is the $Q$-function. We are interested in deriving the PEP by averaging (53) over all possible realizations of $\mathbf{D}^{2}$ :

$$
P_{\mathrm{R}-\mathrm{SM}}(\mathbf{x} \rightarrow \hat{\mathbf{x}})=\mathrm{E}_{\mathbf{D}^{2}}\left[Q\left(\sqrt{\frac{\mathbf{c}^{H} \mathbf{D}^{2} \mathbf{c}}{2} \gamma_{1}}\right)\right] .
$$

Just like SM, in R-SM there are three possible types of symbol errors: i) signal errors denoted as $\mathcal{E}_{1}=\left\{\mathbf{x} \rightarrow \hat{\mathbf{x}} \mid\left\{s_{k} \rightarrow\right.\right.$ $\left.\left.\hat{s}_{k}, \mathbf{e}_{i} \rightarrow \mathbf{e}_{i}\right\}\right\} ;$ ii) space errors denoted as $\mathcal{E}_{2}=\left\{\mathbf{x} \rightarrow \hat{\mathbf{x}} \mid\left\{s_{k} \rightarrow\right.\right.$ $\left.\left.s_{k}, \mathbf{e}_{i} \rightarrow \mathbf{e}_{\hat{i}}\right\}\right\}$; and iii) joint signal and space errors denoted as $\mathcal{E}_{3}=\left\{\mathbf{x} \rightarrow \hat{\mathbf{x}} \mid\left\{s_{k} \rightarrow \hat{s}_{k}, \mathbf{e}_{i} \rightarrow \mathbf{e}_{i}\right\}\right\}$. A signal error occurs when the receiving antenna (represented by $\mathbf{e}_{i}$ ) is correctly detected, while the transmitted symbol $s_{k}$ is incorrectly detected. In contrast, a space error takes place when $s_{k}$ is correctly detected and the receiving antenna is incorrectly detected. Finally, the incorrect detection of $s_{k}$ and $\mathbf{e}_{i}$ creates a joint symbol error. Thus, depending on the type of the R-SM symbol error, the RV $z=\mathbf{c}^{H} \mathbf{D}^{2} \mathbf{c}$ takes the following form:

$z= \begin{cases}\left|s_{k}-\hat{s}_{k}\right|^{2} d_{i}^{2}, & \text { for } \mathcal{E}_{1}, \\ \left|s_{k}\right|^{2}\left(d_{i}^{2}+d_{\hat{i}}^{2}\right), & \text { for } \mathcal{E}_{2}, \\ \left|s_{k}\right|^{2} d_{i}^{2}+\left|\hat{s}_{k}\right|^{2} d_{\hat{i}}^{2}, & \text { for } \mathcal{E}_{3} .\end{cases}$

As we see from (55a)-(55c), $z$ depends on the RVs $d_{i}^{2}$, $i=1, \ldots, N_{R}$. Thus, before continuing with the evaluation of (54), let us provide the p.d.f. of $d_{i}^{2}, i=1, \ldots, N_{R}$, which is fundamental for our proof. For ZF precoding, it holds that:

$$
d_{i}^{2}=\frac{1}{\left[\left(\mathbf{H}_{S R} \mathbf{H}_{S R}^{H}\right)^{-1}\right]_{i, i}} \text {. }
$$

In [48], it is given that $d_{i}^{2} \sim \operatorname{Gamma}(L, 1)$, where $L=N_{t}-$ $N_{R}+1$. Here, $\operatorname{Gamma}(k, \theta)$ denotes a gamma distribution with shape $k$ and scale $\theta$. Thus, the $\operatorname{RVs~} d_{i}^{2}, i=1, \ldots, N_{R}$ have the following p.d.f.:

$$
f_{d_{i}^{2}}(x)=\frac{1}{\Gamma(L)} x^{L-1} e^{-x} H_{0}(x) .
$$

In the following, we evaluate (54) for all different types of R-SM symbol errors. Let us begin with the case of the signal errors. If we set $\delta=s_{k}-\hat{s}_{k}$, plug (55a) into (54), and use (57) 
in order to average over $d_{i}^{2}$, we can bound the PEP of a signal error as:

$$
\begin{aligned}
P_{\mathrm{R}-\mathrm{SM}}\left(\mathbf{x} \rightarrow \hat{\mathbf{x}} \mid \mathcal{E}_{1}\right) & =\mathrm{E}_{d_{i}^{2}}\left[Q\left(\sqrt{\frac{|\delta|^{2} d_{i}^{2}}{2} \gamma_{1}}\right)\right] \\
& \leq \frac{1}{2}\left[\frac{|\delta|^{2}}{4} \gamma_{1}+1\right]^{-L} .
\end{aligned}
$$

In the previous procedure, we use the Chernoff bound $Q(x) \leq$ $(1 / 2) e^{-\left(x^{2} / 2\right)}$ [34]. We note that (58) is also the PEP of a Multiple-Input Single Output (MISO) system with ZF precoding. As it can be seen, this MISO system has a diversity order of $L=N_{t}$ (given that there is one receive antenna). This conclusion agrees with the indirect diversity analysis of [43].

As implied by (55b) and (55c), the evaluation of (54) for the cases of: i) space and ii) joint errors, requires the joint p.d.f. of $d_{i}^{2}$ and $d_{\hat{i}}^{2}$. From (56), it is clear that $d_{i}^{2}$ and $d_{\hat{i}}^{2}$ are correlated RVs. Given that $d_{i}^{2}$ and $d_{\hat{i}}^{2}$ are correlated and distribute as $\operatorname{Gamma}(L, 1)$, their joint p.d.f. is the Kibble's bivariate gamma distribution:

$$
h_{d_{i}^{2}, d_{\hat{i}}^{2}}(x, y)=\frac{\left(1-\rho_{c}\right)^{-L}}{\Gamma(L)} \sum_{k=0}^{+\infty} b_{k} f_{k}(x) p_{k}(y),
$$

as it is given in [49]. Here, we have that:

$$
\begin{aligned}
b_{k} & =\frac{\rho_{c}^{k}}{\left(1-\rho_{c}\right)^{2 k} \Gamma(L+k) k !}, \\
f_{k}(x) & =\left[x^{L+k-1} e^{\frac{-x}{1-\rho_{c}}}\right] H_{0}(x),
\end{aligned}
$$

and

$$
p_{k}(y)=\left[y^{L+k-1} e^{\frac{-y}{1-\rho_{c}}}\right] H_{0}(y) .
$$

In addition, $\rho_{c}=\mathrm{E}\left[\left(d_{i}^{2}-L\right)\left(d_{\hat{i}}^{2}-L\right)\right] / L$, which is the Pearson product-moment correlation coefficient. Given the marginal gamma p.d.f. of each $d_{i}$ and a given system configuration, $\rho_{c}$ can be robustly estimated.

In order to provide a bound on the PEP when a space error occurs, we use (55b) and average (54) over all realizations of $d_{i}^{2}$ and $d_{\hat{i}}^{2}$, using (59). Due to space limitation, we do not provide the details. The attained bound is written as:

$$
\begin{aligned}
P_{\mathrm{R}-\mathrm{SM}}\left(\mathbf{x} \rightarrow \hat{\mathbf{x}} \mid \mathcal{E}_{2}\right) & =\mathrm{E}_{d_{i}^{2}, d_{\hat{i}}^{2}}\left[Q\left(\sqrt{\frac{\left|s_{k}\right|^{2}\left(d_{i}^{2}+d_{\hat{i}}^{2}\right)}{2} \gamma_{1}}\right)\right] \\
& \leq \frac{\left(1-\rho_{c}\right)^{-L}}{2 t^{2 L}}{ }_{1} F_{0}\left(L ; ; \frac{\rho_{c}}{\left(1-\rho_{c}\right)^{2} t^{2}}\right),
\end{aligned}
$$

where $t=\left(\left|s_{k}\right|^{2} \gamma_{1} / 4\right)+\left(1 /\left(1-\rho_{c}\right)\right)$ and

$$
{ }_{1} F_{0}(L ; ; y)=\sum_{k=0}^{+\infty} \frac{[L]_{k}}{k !} y^{k}
$$

is the generalized hypergeometric function, as defined in [41, p. 1010]. Moreover, $[L]_{k}=L(L+1) \ldots(L+k-1)$ is the Pochhammer symbol.

Similarly, the bound on the PEP of the joint error of $\mathcal{E}_{3}$ is achieved by evaluating the expectation of (54), using (55c) and (59):

$$
\begin{aligned}
& P_{\mathrm{R}-\mathrm{SM}}\left(\mathbf{x} \rightarrow \hat{\mathbf{x}} \mid \mathcal{E}_{3}\right) \\
& =\mathrm{E}_{d_{i}^{2}, d_{\hat{i}}^{2}}\left[Q\left(\sqrt{\frac{\left|s_{k}\right|^{2} d_{i}^{2}+\left|\hat{s}_{k}\right|^{2} d_{\hat{i}}^{2}}{2} \gamma_{1}}\right)\right] \\
& \leq \frac{\left[\left(1-\rho_{c}\right) t_{1} t_{2}\right]^{-L}}{2} \times{ }_{1} F_{0}\left(L ; ; \frac{\rho_{c}}{t_{1} t_{2}\left(1-\rho_{c}\right)^{2}}\right),
\end{aligned}
$$

where $t_{1}=\left(\left|s_{k}\right|^{2} \gamma_{1} /\right)+\left(1 /\left(1-\rho_{c}\right)\right)$ and $t_{2}=\left(\left|\hat{s}_{k}\right|^{2} \gamma_{1} / 4\right)+$ $\left(1 /\left(1-\rho_{c}\right)\right)$.

As a final step for our proof, we employ the bounds of (58), (63), and (65), and express the ABEP of the first hop as:

$$
P_{1}\left(\gamma_{1}\right) \leq \frac{1}{M k_{t}} \sum_{\mathbf{x}} \sum_{\mathbf{x} \neq \hat{\mathbf{x}}} d(\mathbf{x} \rightarrow \hat{\mathbf{x}}) P_{\mathrm{R}-\mathrm{SM}}(\mathbf{x} \rightarrow \hat{\mathbf{x}}),
$$

where $d(\mathbf{x} \rightarrow \hat{\mathbf{x}})$ denotes the number of different bits between the bit sequences represented by $\mathrm{x}$ and $\hat{\mathrm{x}}$.

In order to complete the derivation of (52), we express the ABEP of the second hop using the results from [46]. Hence, the ABEP of the second hop is bounded as:

$$
P_{2}\left(\gamma_{2}\right) \leq \frac{1}{M k_{t}} \sum_{\mathbf{x}} \sum_{\mathbf{x} \neq \hat{\mathbf{x}}} d(\mathbf{x} \rightarrow \hat{\mathbf{x}}), P_{\mathrm{SM}}(\mathbf{x} \rightarrow \hat{\mathbf{x}}),
$$

where

$$
P_{\mathrm{SM}}(\mathbf{x} \rightarrow \hat{\mathbf{x}})=\frac{1}{2} \operatorname{det}\left(\mathbf{I}_{\zeta, \zeta}+\gamma_{2} \frac{\Lambda}{\sqrt{2}}\right) .
$$

Here, we have that $\zeta=N_{D} N_{R}$ and $\Lambda=\mathbf{I}_{N_{D}, N_{D}} \otimes \mathbf{c c}^{H}$.

\section{B. Average Bit Error Probability When the RNs Employ Distributed Detection}

This section provides the ABEP of DH-HSSK/HSM when the RNs utilize the DD of (51). In this type of uncoordinated detection, the methodology that we follow in the previous subsection does not hold, because during the second hop multiple RNs can be active. In this case, the transmitted symbol vector of the second hop is not a legal SM symbol. Thus, (52) is not valid.

In order to overcome this problem, we use the union bound technique and express the end-to-end ABEP of DH-HSM as:

$P_{\mathrm{D}}\left(\gamma_{1}, \gamma_{2}\right) \leq \frac{1}{M k_{t}} \sum_{\mathbf{x}} \sum_{\mathbf{x} \neq \hat{\mathbf{x}}} d(\mathbf{x} \rightarrow \hat{\mathbf{x}}) P_{\mathrm{DD}}\left(\mathbf{x} \rightarrow \hat{\mathbf{x}}, \gamma_{1}, \gamma_{2}\right)$,

where $P_{\mathrm{DD}}\left(\mathbf{x} \rightarrow \hat{\mathbf{x}}, \gamma_{1}, \gamma_{2}\right)$ is the end-to-end PEP of transmitting $\mathrm{x}$ at the $\mathrm{SN}$ and detecting erroneously $\hat{\mathrm{x}} \neq \mathrm{x}$ at the 
$\mathrm{DN}$, given $\gamma_{1}$ and $\gamma_{2}$ (the SNR of each hop). We remind that $\mathbf{x}, \hat{\mathbf{x}} \in \mathcal{A}_{1}=\left\{\mathbf{e}_{i} s_{k} \mid i \in\left\{1, \ldots, N_{R}\right\} \cap k \in\{1, \ldots, M\}\right\}$.

It is not difficult to see that, using the total probability theorem, the PEP of an end-to-end symbol error is expressed as:

$$
P_{\mathrm{DD}}\left(\mathbf{x} \rightarrow \hat{\mathbf{x}}, \gamma_{1}, \gamma_{2}\right)=\sum_{\tilde{\mathbf{x}} \in \mathcal{A}} P_{\mathrm{SR}}\left(\mathbf{x} \rightarrow \tilde{\mathbf{x}}, \gamma_{1}\right) P_{\mathrm{RD}}\left(\tilde{\mathbf{x}} \rightarrow \hat{\mathbf{x}}, \gamma_{2}\right)
$$

where $\tilde{\mathbf{x}} \in \mathcal{A}$ are all the possible detected/transmitted symbol vectors at the RNs. $P_{\mathrm{SR}}\left(\mathbf{x} \rightarrow \tilde{\mathbf{x}}, \gamma_{1}\right)$ is the probability that $\mathbf{x}$ is transmitted at the $\mathrm{SN}$ and $\tilde{\mathrm{x}}$ is detected/retransmitted at the RNs. Furthermore, $P_{\mathrm{RD}}\left(\tilde{\mathbf{x}} \rightarrow \hat{\mathbf{x}}, \gamma_{2}\right)$ represents the probability that $\tilde{\mathbf{x}}$ is transmitted at the RNs and $\hat{\mathbf{x}}$ is detected at the DN.

Before continuing, we define the set $\mathcal{A}=$ $\left\{\mathcal{A}_{0}, \mathcal{A}_{1}, \ldots, \mathcal{A}_{N_{R}}\right\}$. Here, we have that $\mathcal{A}_{0}=\left\{\boldsymbol{0}_{N_{R}, 1}\right\}$. The subset $\mathcal{A}_{0}$ corresponds to the very special case where all the RNs remain silent during the second hop (for more details, the reader is referred to Section IV). Every other subset $\mathcal{A}_{q}$, with $q=1, \ldots, N_{R} \quad$ and $\quad\left|\mathcal{A}_{q}\right|=\left(\begin{array}{c}N_{R} \\ q\end{array}\right) M^{q}$, contains all the possible vectors, of size $N_{R} \times 1$, with exactly $q$ non zero elements. Their non zero elements take values from $\mathcal{M}=\left\{s_{1}, \ldots, s_{k}\right\}$. Mathematically, this is represented as $\mathcal{A}_{q}=\left\{\mathbf{e}_{i_{1}} s_{k_{1}}+\cdots+\mathbf{e}_{i_{q}} s_{k_{q}} \mid \mathcal{B}_{1} \cap \mathcal{B}_{2}\right\}$, where $\mathcal{B}_{1}=\left\{\left\{i_{1}, \ldots, i_{q}\right\} \in\left\{1, \ldots, N_{R}\right\} \mid\left\{i_{1} \neq \cdots \neq i_{q}\right\}\right\}$, $\mathcal{B}_{2}=\left\{\left\{k_{1}, \ldots, k_{q}\right\} \in\{1, \ldots, M\}\right\}$, and $\mathbf{e}_{i_{l}}$ is the $i_{l}$-th column of $\mathbf{I}_{N_{R}, N_{R}}$. We note that, clearly, the correct symbol $\mathbf{x}$ belongs to $\mathcal{A}$. Even thought the RNs might be able to detect correctly the transmitted vector $\mathbf{x}$, a symbol error could occur during the second hop.

We have to evaluate each probability of the right hand side of (70). We begin with $P_{\mathrm{SR}}\left(\mathbf{x} \rightarrow \tilde{\mathbf{x}}, \gamma_{1}\right)$. Given that detection is conducted independently at each RN and the Gaussian noise is independent, we have:

$$
P_{\mathrm{SR}}\left(\mathbf{x} \rightarrow \tilde{\mathbf{x}}, \gamma_{1}\right)=\prod_{j=1}^{N_{R}} \mathrm{E}_{d_{j}^{2}}\left[P_{\mathrm{SR}_{j}}\left(\mathbf{x}_{j} \rightarrow \tilde{\mathbf{x}}_{j}, \gamma_{1} \mid d_{j}^{2}\right)\right]
$$

Here, $\mathbf{x}_{j}, \tilde{\mathbf{x}}_{j} \in\left\{s_{0}, \mathcal{M}\right\}$ are the $j$-th elements of $\mathbf{x}$ and $\tilde{\mathbf{x}}$, respectively. In simple words, $\tilde{\mathbf{x}}_{j}$ belongs to the employed constellation $\mathcal{M}$ or is 0 and $\tilde{\mathbf{x}}_{j}$ is the detected symbol which again belongs to $\mathcal{M}$ or is zero.

Thus, we have to evaluate

$$
P_{\mathrm{SR}_{\mathrm{j}}}\left(\mathbf{x}_{j} \rightarrow \tilde{\mathbf{x}}_{j}, \gamma_{1}\right)=\mathrm{E}_{d_{j}^{2}}\left[P_{\mathrm{SR}_{j}}\left(\mathbf{x}_{j} \rightarrow \tilde{\mathbf{x}}_{j}, \gamma_{1} \mid d_{j}^{2}\right)\right]
$$

by integrating over all the possible realizations $d_{j}^{2}$. The received signal at the $j$-th RN is given from (6). Hence, given that $\mathbf{x}_{j}, \tilde{\mathbf{x}}_{j} \in\left\{s_{0}, \mathcal{M}\right\}$, with $\mathbf{x}_{j} \neq \tilde{\mathbf{x}}_{j}$, we can write

$$
P_{\mathrm{SR}_{j}}\left(\mathbf{x}_{j} \rightarrow \tilde{\mathbf{x}}_{j}, \gamma_{1} \mid d_{j}^{2}\right)=Q\left(\sqrt{\frac{\gamma_{1}\left|\mathbf{x}_{j}-\tilde{\mathbf{x}}_{j}\right|^{2} d_{j}^{2}}{2}}\right) .
$$

For this reason, if we follow the same steps as the derivation of (58), we can prove that:

$$
P_{\mathrm{SR}_{j}}\left(\mathbf{x}_{j} \rightarrow \tilde{\mathbf{x}}_{j}, \gamma_{1}\right) \leq \frac{1}{2}\left[\frac{\left|\mathbf{x}_{j}-\tilde{\mathbf{x}}_{j}\right|^{2}}{4} \gamma_{1}+1\right]^{-L}
$$

For the case of $\mathbf{x}_{j}=\tilde{\mathbf{x}}_{j}$ (correct transmission to the $j$-th $\mathrm{RN}$ ), we have that

$$
\begin{aligned}
P_{\mathrm{SR}_{j}}\left(\mathbf{x}_{j} \rightarrow \tilde{\mathbf{x}}_{j}, \gamma_{1}\right) & =1-P_{e}\left(\gamma_{1}\right) \\
& \leq 1-\sum_{\substack{j=0 \\
\mathbf{x}_{j} \neq s_{j}}}^{M} P_{\mathrm{SR}_{j}}\left(\mathbf{x}_{j} \rightarrow s_{j}, \gamma_{1}\right),
\end{aligned}
$$

where $P_{e}\left(\gamma_{1}\right)$ is the probability of erroneous detection. Hence, the probability of interest is bounded by

$$
P_{\mathrm{SR}_{j}}\left(\mathbf{x}_{j} \rightarrow \tilde{\mathbf{x}}_{j}, \gamma_{1}\right) \leq 1-\frac{1}{2} \sum_{\substack{j=0 \\ x_{j} \neq s_{j}}}^{M}\left[\frac{\left|\mathbf{x}_{j}-s_{j}\right|^{2}}{4} \gamma_{1}+1\right]^{-L} .
$$

In addition, the evaluation of (70) requires the knowledge of $P_{\mathrm{RD}}\left(\tilde{\mathbf{x}} \rightarrow \hat{\mathbf{x}}, \gamma_{2}\right)$. We know that $\tilde{\mathbf{x}} \in \mathcal{A}$, which means that the RNs retransmit legal or illegal SM symbols. Even in the case of the retransmission of illegal SM symbols, as we explain in Section IV, the DN uses the detector of (9). Thus an error happens when $\mathcal{E}^{\prime}=\left\{\left\|\mathbf{y}_{D}-\mathbf{H}_{R D} \mathbf{x}\right\|_{2}^{2}>\left\|\mathbf{y}_{D}-\mathbf{H}_{R D} \hat{\mathbf{x}}\right\|_{2}^{2} \mid \tilde{\mathbf{x}}\right\}$ (given that $\tilde{\mathbf{x}}$ is relayed by the $\mathrm{RNs}$ ). We can easily transform $\mathcal{E}^{\prime}$ as $\mathcal{E}^{\prime}=\left\{\left\|\mathbf{H}_{R D} \mathbf{c}_{1}+\mathbf{w}_{R D}\right\|_{2}^{2}>\left\|\mathbf{H}_{R D} \mathbf{c}_{2}+\mathbf{w}_{R D}\right\|_{2}^{2}\right\}$, where $\mathbf{c}_{1}=\tilde{\mathbf{x}}-\mathbf{x}$ and $\mathbf{c}_{2}=\tilde{\mathbf{x}}-\hat{\mathbf{x}}$. We can further proceed and reach to $\mathcal{E}^{\prime}=\left\{\left\|\mathbf{H}_{R D} \mathbf{c}_{1}\right\|_{2}^{2}-\left\|\mathbf{H}_{R D} \mathbf{c}_{2}\right\|_{2}>2 \operatorname{Re}\left\{\mathbf{c}^{H} \mathbf{H}_{R D}^{H} \mathbf{w}_{R D}\right\}\right\}$, where $\mathbf{c}=\mathbf{c}_{2}-\mathbf{c}_{1}$. Given that $-\sum_{k=1}^{N D} \operatorname{Re}\left\{\mathbf{w}_{R D_{k}} a_{k}\right\} \sim$ $\mathcal{N}\left(0,\left(\sigma_{w_{R D}}^{2} / 2\right)\left\|\mathbf{H}_{R D} \mathbf{c}\right\|_{2}^{2}\right)$, where $a_{k}=\sum_{j=1}^{N_{D}} \mathbf{c}_{j}^{*}\left[\mathbf{H}_{R D}\right]_{j, k}^{*}$, we have that the instantaneous probability for which we are interested is written as:

$$
P_{\mathrm{RD}}\left(\tilde{\mathbf{x}} \rightarrow \hat{\mathbf{x}}, \gamma_{2} \mid \mathbf{H}_{R D}\right)=Q\left(\gamma^{\prime} \frac{\left\|\mathbf{H}_{R D} \mathbf{c}_{2}\right\|_{2}^{2}-\left\|\mathbf{H}_{R D} \mathbf{c}_{1}\right\|_{2}^{2}}{\sqrt{\left\|\mathbf{H}_{R D} \mathbf{c}\right\|_{2}^{2}}}\right),
$$

where $\gamma^{\prime}=\sqrt{\gamma_{2} / 2}$. The direct evaluation of the expectation of (77), over all channel realizations, is a difficult task. Actually, this expectation requires the p.d.f. of the summation of two Erlang (gamma) RVs divided by a generalized gamma $\mathrm{RV}$, where all RVs are correlated. An approach that gives a tractable solution to the expectation of (77) is to use the triangle and inverse triangle inequality and upper and lower bound the RV $Y=\left(\left\|\mathbf{H}_{R D} \mathbf{c}_{2}\right\|_{2}^{2}-\left\|\mathbf{H}_{R D} \mathbf{c}_{1}\right\|_{2}^{2}\right) / \sqrt{\left\|\mathbf{H}_{R D} \mathbf{c}\right\|_{2}^{2}}$. Unfortunately, this method gives bounds that are loose and do not provide any insight. Due to space limitation, we do not include this analysis. An alternative way to evaluate the expectation of (77) is numerically. In this way, we conclude our proof.

\section{Simulation Results and Discussion}

In this section, we present theoretical and Monte Carlo simulation results that demonstrate the performance of the proposed DH-HSSK/HSM architecture under different forms of CSIT at the SN, both for the CD and DD algorithms. We consider scenarios with: i) small, ii) medium and iii) large system configuration.

For the sake of comparison, we compare our architecture against four benchmark systems: i) the corresponding single 
relay system, ii) the best relay selection system, iii) a form of distributed Alamouti relaying, and iv) the direct communication link. We note that in all the benchmark systems, the operating principle of the $\mathrm{SN}$ and the DN is the same as DH-HSM/HSSK. The SN uses the same linear precoding methods and the DN detects the received signal using ML detection.

The corresponding single relay system is denoted as Dual Hop-with one Relay (DH-w1R). The adoption of this form of comparison for the evaluation of space modulated VMIMO is extensively employed in the literature [20], [24], [27], [28], [32]. The operational principle of DH-W1R is similar to the proposed DH-HSSK/HSM architecture with the following exception. The conveyed constellation point is relayed by a single RN.

We note that the $\mathrm{RN}$ of $\mathrm{DH}-\mathrm{w} 1 \mathrm{R}$ detects the relayed constellation point using a ML detector which has almost the same complexity as the ML detector of (7) and higher complexity than the detector of (51) (all detectors are single stream detectors). Especially, the DD of (51) results in a significant lower complexity as the spectral efficiency and the number of the RNs is increased. Because of this, DH-HSSK/HSM can be interpreted as a way of distributing the computational complexity of the single RN of DH-W1R to the multiple RNs of DH-HSSK/HSM. An additional advantage of DH-HSSK/HSM compared with DH-W1R is that, under the assumption of high SNR at the RNs, usually only one out of $N_{R}$ RNs is retransmitting the symbol received from the SN. This is very important when the RNs are remote terminals (for example mobile phones) with a battery supply, because a battery life extension is achieved. ${ }^{1}$ Hence, if we take into account the advantages of DH-HSSK/HSM compared with DH-W1R with respect to: i) the complexity and power consumption distribution and ii) the BER gains (as shown in the following), it is clear that the cost of having multiple RNs of low complexity and extended battery life compared with the cost of having a single RN of high complexity is insignificant.

The next two benchmark systems that we employ is the: i) best relay selection technique denoted as Dual Hop-with Best Relay Selection (DH-wBRS) and ii) the distributed Alamouti code represented as Dual Hop-Distributed Alamouti Code (DHDAC). In DH-wBRS, a single RN out of $N_{R}$ is selected to convey the transmitted constellation point in every block of symbols. DH-wBRS selects the RN with the most favorable RN-DN channel which has the highest channel gain. The selection of this RN offers the highest instantaneous receive SNR at the DN. In DH-DAC, two symbols are conveyed to two RNs in order to form a distributed Alamouti codeword. The RNs can employ a CD or DD algorithm, just like DH-HSM. In the DD case, the orthogonality of the Alamouti codeword cannot be ensured, whereas in the $\mathrm{CD}$ case it can be ensured. We note that DH-DAC requires 4 symbol period in order to convey two constellation points (constructed Alamouti codeword) from the SN to the DN. Clearly, the comparison of DH-HSM with DHwBRS and DH-DAC is not entirely fair. DH-wBRS uses closed

\footnotetext{
${ }^{1}$ In this paper, we assume that the energy consumption for the RF power transmission is relatively high compared to the energy consumption of the circuits of the RNs during the detection period.
}

loop transmission during the second hop, while DH-HSM uses open loop transmission. Furthermore, DH-DAC activates two RNs during the second hop, whereas DH-HSM activate only one. Finally, we state that: i) DH-DAC is used as a benchmark system only when the RNs are two and ii) no best RN selection criterion is applied in DH-DAC. As we see in the following, even though these comparisons are not entirely fair, DH-HSM is able to achieve significant performance gains in the majority of the cases.

The last benchmark system that we use is the direct SN-DN MIMO communication. We assume that the SN-DN wireless channel is weak and distributes as $\mathcal{C N}(\mathbf{0}, p \mathbf{I})$, where $p$ captures the path loss effect. This assumption is valid because DH-HSM is designed to be applied when the SN-DN channel is weak.

For all systems, the SNR during the first hop is defined as $\gamma_{1}=\mathrm{SNR}_{\mathrm{dB}}^{\mathrm{SR}}=10 \log _{10}\left(P_{s}^{S R} / \sigma_{w_{S R}}^{2}\right)$, where $P_{s}^{S R}=$ $\mathrm{E}_{\mathbf{x}}\left[\|\mathbf{P D x}\|_{2}^{2}\right]$ is the power transmitted at SN. For a fair comparison between DH-HSSK/HSM and DH-w1R, we set $P_{s}=1$ by using the appropriate: i) normalization matrix $\mathbf{D}$ for the precoder $\mathbf{P}$ (as described in Section II) and ii) the appropriate normalization of the conventional transmitted constellation diagram. During the second hop, the SNR is defined as $\gamma_{1}=\mathrm{SNR}_{\mathrm{dB}}^{\mathrm{RD}}=10 \log _{10}\left(P_{s}^{R D} / \sigma_{w_{R D}}^{2}\right)$, where $P_{s}^{R D}=1$ is the transmitted power from the active RN. Without loss of generality we assume that $\gamma_{1}=\gamma_{2}$. Finally, we assume that the SN-RNs and RNs-DN wireless channel remain constant over a block symbol. In addition, when I-CSIT is available at the SN, $\overline{\mathbf{H}}_{\mathrm{SR}}$ does not change over a block of symbols.

\section{A. Confirmation of the Average Bit Error Probability of DH-HSM Using Simulation Results}

Before we proceed with the comparison of DH-HSM with the benchmark system, in Fig. 2, we demonstrate how close are the obtained simulation results with the theoretical framework of Section V. Fig. 2(a) presents the bound of ABEP when $\mathrm{CD}$ is employed at the RNs. In addition, Fig. 2(b) shows the ABEP bound under DD at the RNs. We consider two system configurations for $\mathrm{CD}$. In the first configuration, we have a $8 \times$ $4 \times 4\left(N_{t}=8, N_{R}=4\right.$, and $\left.N_{D}=4\right)$ system with $k_{t}=1 \mathrm{bpsp}$ for DH-HSSK and $k_{t}=2$ bpsp for DH-HSM. Furthermore, the second configuration is a $16 \times 8 \times 4$ system with $k_{t}=$ 1.5 bpsp for DH-HSSK and $k_{t}=2.5$ bpsp for DH-HSM. As can be seen from Fig. 2(a), for CD and in high SNR, the theoretical and simulation curves perfectly match. In low SNR, there is a difference which is a well known phenomenon caused by the union bound technique [34]. In addition, Fig. 2(b), demonstrates that the bound obtained for DD in Section V-B is close to the simulated curves.

\section{B. Small Scale System Setup}

Fig. 3 explores the BER performance of DH-HSM when P-CSIT is available at the DN and the system setup is $4 \times 2 \times$ 4 , with $k_{t}=4$ bpsp. For the sake of comparison, in Fig. 3, we include the performance of: i) DH-w1R, ii) DH-wBRS, iii) DHDAC, and iii) direct link communication when $p=\{0.1,1\}$. Especially, the scenario of the direct link communication with 


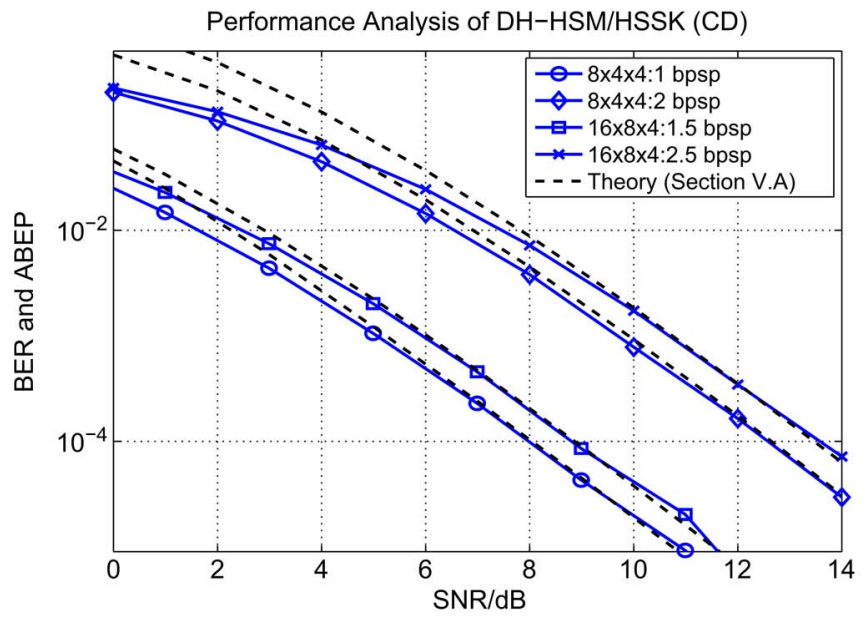

(a)

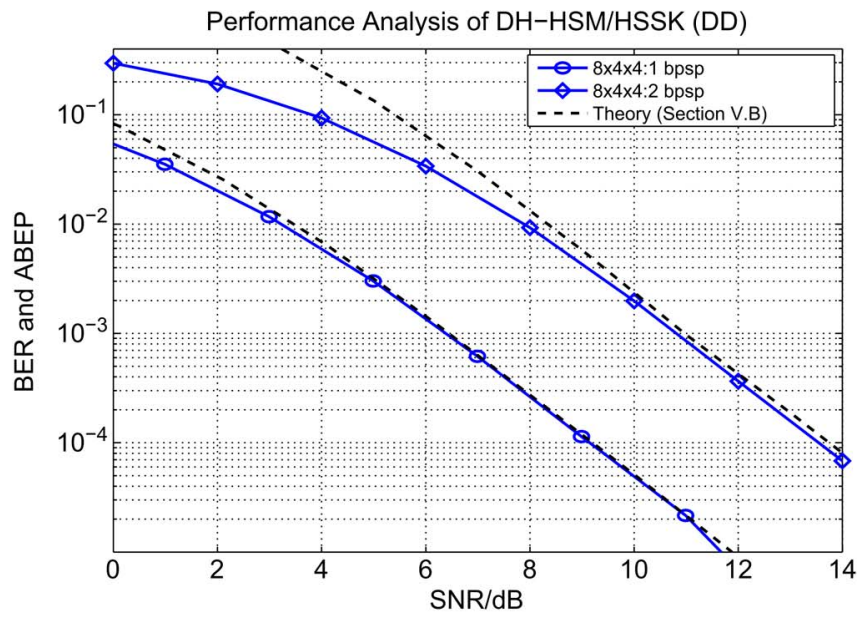

(b)

Fig. 2. Performance analysis of DH-HSM when ZF with P-CSIT is employed at the RNs, using: i) simulation results and ii) the bounds of Section V. (a) The RNs employ the CD algorithm. (b) The RNs employ the DD algorithm.

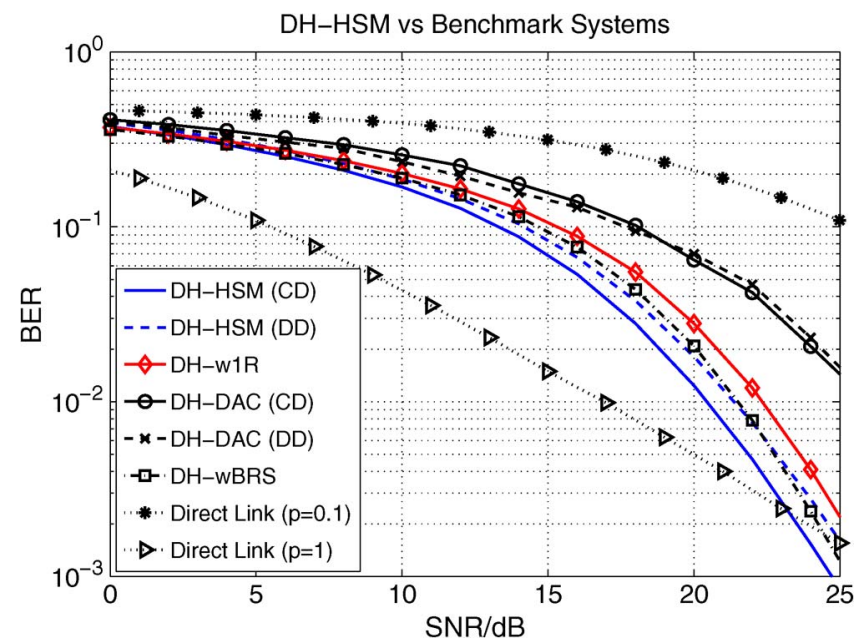

Fig. 3. BER performance of a small scale DH-HSM $(4 \times 2 \times 4)$ system versus DH-w1R, DH-wBRS, DH-DAC, and the $4 \times 4$ direct link communication when $p=\{0.1,1\}$. The spectral efficiency is $k_{t}=4 \mathrm{bpsp}$.

a weak SN-DN channel $(p=0.1)$ is a typical application scenario of DH-HSM (or any other dual hop system). Furthermore, as a reference point, we include the performance of direct link communication when the SN-DN channel is strong with $p=1$ (same statistics as the SN-RNs and RNs-DN channels).

As it can be seen from Fig. 3, DH-HSM offers better BER performance than DH-w1R, DH-wBRS, and DH-DAC due to its multiplexing gain. It quite notable that in the depicted SNR region, DH-HSM offers better performance than DH-wBRS and DH-DAC. Even though, this comparison is not entirely fair. We note that as SNR increases to extremely high value, DH-wBRS and DH-DAC would offer better performance, due to their diversity achieving techniques employed during the second hop. We note that, just like DH-HSM, the performance of DH-DAC is not affected by the employment of CD or $\mathrm{DD}$ at the RNs. Regarding the performance of the direct link communication, we see that when the SN-DN channel is weak $(p=0.1)$, its performance is significantly worse (as expected). For a strong SN-DN channel $(p=1)$, we see that the direct link offers better performance due to its multiplexing gain. Though, in high SNR, the direct link is remarkably outperformed due to its unity diversity order [43].

\section{Medium Scale Setup: The Effect of Multiple Antennas at the $D N$}

The performance evaluation of DH-HSSK/HSM is presented in Fig. 4. In the same figure, we depict the performance of DH-w1R and DH-wBRS for the purpose of comparison. We note that in the following results, we do not use the direct link communication and distributed STBC as benchmark systems. We do not employ the direct link communication, because we propose DH-HSM for a scenario where either the SN-DN is very weak (where direct link communication is clearly outperformed), or there is no SN-DN link. Moreover, distributed STBC is not further used, because as the size of the distributed STBC codeword increases, its fractional STBC rate determines performance significantly worse than DH-HSM.

In addition, we note that for DH-W1R and DH-wBRS, the BER performance is depicted only for $\mathrm{ZF}$ precoding at the SN. It can be proved that in the very special case of a MISO linear precoding system (first hop) and when the normalization process of Section II is applied, the MMSE and the ZF forcing precoders result in the same receiving signal. Hence, the performance is the same.

Fig. 4(a) and (b) demonstrate that when the number of receive antennas is small $N_{D}=\{1,2\}$, there is no performance difference between the $\mathrm{CD}$ and $\mathrm{DD}$ algorithms of DH-HSSK/HSM. As $N_{D}$ is increased, there is a difference. For $N_{D}=4$, the difference can be noticed only in low SNRs. Though, when $N_{D}=16$, this performance gap is significantly increased. This performance difference exists due to the lost multiplexing gain which is caused by the activation of multiple RNs.

Regarding the comparison with the benchmark systems, we see that for $N_{D}>1$, DH-HSSK/HSM is significantly better than DH-w1R. When, $N_{D}=1$, DH-HSSK offer a slightly better performance than DH-w1R. Moreover, DH-HSM is 


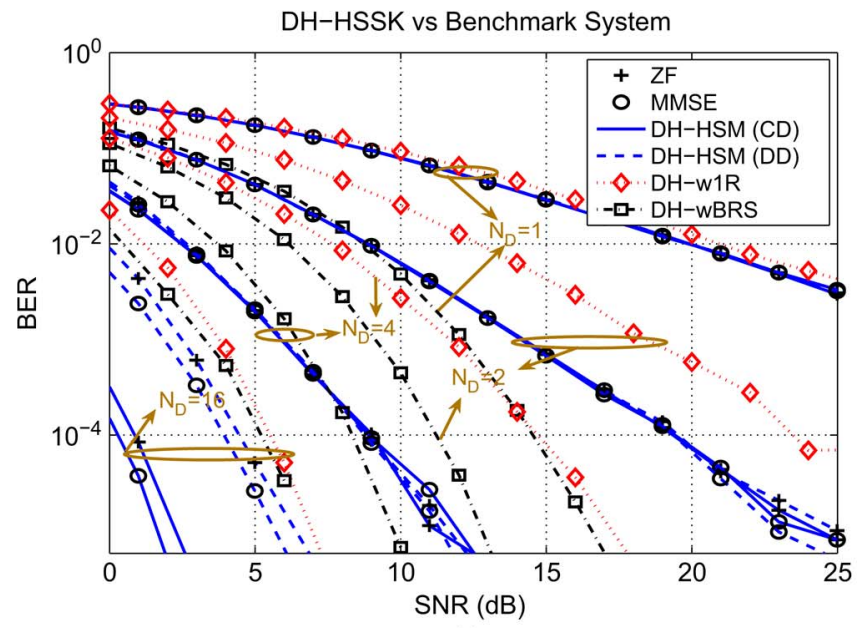

(a)

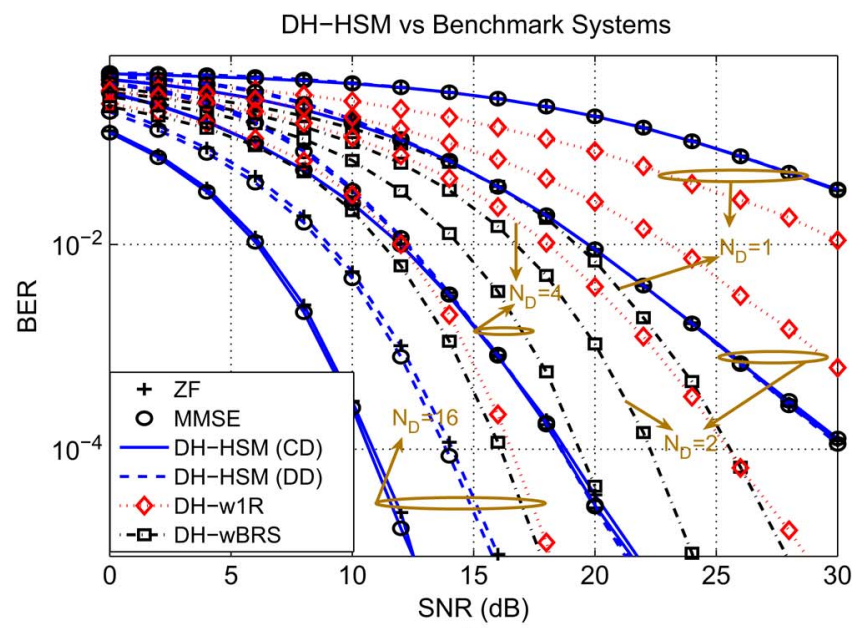

(b)

Fig. 4. BER performance of a medium scale DH-HSSK/HSM system, for $N_{D}=\{1,2,4,16\}$, when: i) the SN employs P-CSIT and ii) the SNR per hop is the same. (a) $16 \times 8 \times N_{D}$ DH-HSSK system $\left(k_{t}=1.5 \mathrm{bpsp}\right)$. (b) $16 \times 8 \times N_{D}$ DH-HSM system $\left(k_{t}=3.5 \mathrm{bpsp}\right)$.

out-performed due to the unity diversity order of SM during the second hop [50].

In addition, Fig. 4(a) and (b) include the comparison of DHHSSK/HSM with DH-wBRS. We recall that this comparison is not fair for DH-HSSK/HSM. For $N_{D}=\{1,2\}$, DH-wBRS demonstrates better performance. In contrast, for a value of $N_{D}=4$ and low SNRs, DH-HSSK/HSM outperforms DHwBRS due to its higher multiplexing gain. Though, after a certain point $(\sim 7.5 \mathrm{~dB}$ and $\sim 20 \mathrm{~dB}$ for DH-HSSK and DHHSM, respectively), DH-wBRS has better performance due to its higher diversity order. Remarkably, when $N_{D}=16$ and for the depicted practical BER, DH-HSSK and DH-HSM offer a significantly better performance, even for DD.

\section{A Very Large Scale Setup}

Recently, the concept of very large scale MIMO (or Massive MIMO) has attracted a great attention from the research community [51]. Thus, in Fig. 5, we explore the performance of DH-HSSK/HSM in a system with a large number of antennas at the $\mathrm{SN}$ and DN, and a large number of RNs $(64 \times 32 \times 64)$.

The first conclusion drawn from Fig. 5 is that both DHHHSK and DH-HSM achieve practical BERs in very low SNRs. For example, DH-HSSK achieves a BER of $10^{-4}$ at about $-5.4 \mathrm{~dB}$ when $\mathrm{CD}$ is employed and at about $-2.2 \mathrm{~dB}$ when DD is employed. This phenomenon is attributed to the massive MIMO setup of the first hop [51] and to the massive multiplexing gain and receive diversity obtained from SM during the second hop. In addition, Fig. 5 demonstrates that the employment of ZF or MMSE precoding at the SN results in a performance gap for both DH-HSSK and DH-HSM architectures, and for both CD and DD. Regarding the use of $\mathrm{CD}$ or DD at the RNs, we observe that there is a penalty for both DH-HSSK and DH-HSM when DD is employed. This penalty is decreased as the value of SNR is decreased.

Furthermore, Fig. 5 shows that DH-HSSK $\left(k_{t}=2.5 \mathrm{bpsp}\right)$ and DH-HSM $\left(k_{t}=4.5 \mathrm{bpsp}\right)$, both for CD and DD, have a significantly better performance than DH-w1R and DH-wBRS,

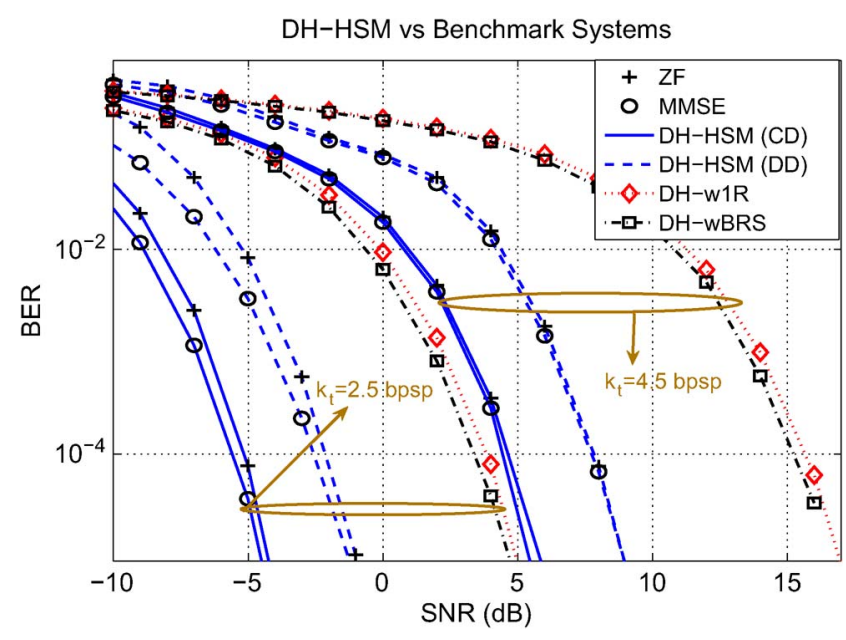

Fig. 5. BER performance of a very large scale DH-HSSK/HSM $(64 \times 32 \times$ 64) system when: i) the SN employs P-CSIT, and ii) the SNR per hop is the same.

for the depicted practical values of BER. We remind the reader that the comparison of DH-HSSK/HSM and DH-wBRS is not entirely fair, due to the closed loop transmission of the second hop of DH-wBRS. It is quite notable, that for $\mathrm{BER}=10^{-4}$, DH-HSSK has a performance difference of about $8 \mathrm{~dB}$ when $\mathrm{CD}$ is employed and $5 \mathrm{~dB}$ when $\mathrm{DD}$ is employed at the RNS, compared with DH-wBRS. The performance difference against DH-w1R is further increased to about $9 \mathrm{~dB}$ when $\mathrm{CD}$ is employed and $6 \mathrm{~dB}$ when DD is employed. Additionally, for the same BER, DH-HSM demonstrates a performance gap of about $9 \mathrm{~dB}$ for $\mathrm{CD}$ and about $6 \mathrm{~dB}$ for $\mathrm{DD}$, compared with $\mathrm{DH}$ wBRS. However, when DH-HSM is compared with DH-w1R, the performance difference is further increased to about $10.5 \mathrm{~dB}$ for $\mathrm{CD}$ and to $7.5 \mathrm{~dB}$ for $\mathrm{DD}$. We note that all systems are able to achieve a huge receive diversity gain due to the big number of receive antennas at the DN.

At this point, we note that due to the simplicity, low complexity, and very good BER performance of DH-HSSK/HSM at very low SNRs, our architecture would be a perfect candidate 
TABLE I

RELATIVE ENERGY EFFICIENCY GAINS OF DH-HSSK/HSM VERSUS DH-W1R FOR DIFFERENT SYSTEM CONFIGURATIONS

\begin{tabular}{c|c|c|c|c}
\hline \hline \multicolumn{2}{c|}{ Configuration } & $k$ (bpsp) & Target BER & RAER [\%] \\
\hline \hline DH-HSSK & $8 \times 4 \times 1$ & 1 & $10^{-2}$ & $0 \%$ \\
with CD & $16 \times 8 \times 4$ & 1.5 & $10^{-3}$ & $77.6 \%$ \\
at the RNs & $64 \times 32 \times 64$ & 2.5 & $10^{-4}$ & $87 \%$ \\
\hline DH-HSSK & $8 \times 4 \times 1$ & 1 & $10^{-2}$ & $0 \%$ \\
with DD & $16 \times 8 \times 4$ & 1.5 & $10^{-3}$ & $77.6 \%$ \\
at the RNs & $64 \times 32 \times 64$ & 2.5 & $10^{-4}$ & $75 \%$ \\
\hline \hline DH-HSM & $8 \times 4 \times 1$ & 3 & $10^{-1}$ & $-14.5 \%$ \\
with CD & $16 \times 8 \times 4$ & 3.5 & $10^{-3}$ & $77.6 \%$ \\
at the RNs & $64 \times 32 \times 64$ & 4.5 & $10^{-4}$ & $91 \%$ \\
\hline DH-HSM & $8 \times 4 \times 1$ & 3 & $10^{-1}$ & $-14.5 \%$ \\
with DD & $16 \times 8 \times 4$ & 3.5 & $10^{-3}$ & $77.6 \%$ \\
at the RNs & $64 \times 32 \times 64$ & 4.5 & $10^{-4}$ & $82.2 \%$ \\
\hline \hline
\end{tabular}

for wireless backhaul connection between BSs with a very large number of antennas. Such an application could be easily implemented using either fixed or mobile RNs, or a combination of them.

\section{E. RF Energy Efficiency Gains of DH-HSSK/HSM}

In order to quantify the RF energy efficiency gain of DHHSSK/HSM over the benchmark system of DH-W1R, we employ the metric of the Relative Average Energy Reduction (RAER) defined as:

$$
\operatorname{RAER}[\%]=\left[1-10^{-\frac{\Delta_{\mathrm{SNR}}}{10}}\right] \times 100 \% .
$$

$\Delta_{\text {SNR }}$ denotes the SNR (in $\mathrm{dB}$ ) difference between DHHSSK/HSM and DH-W1R for a given target BER.

Table I demonstrates the RAER benefits of DH-HSSK/HSM over DH-W1R, for different system configurations and spectral efficiency (in bpsp). In order to conduct a fair comparison between DH-HSSK/DH-HSM and the benchmark system, we use the same: i) BER target and ii) spectral efficiency, for each system configuration. As it can be seen from Table I, in almost all studied scenarios, our DH-HSSK and DH-HSM architectures are more energy efficient than DH-W1R. There are only two exceptions, when the DN is equipped with one receive antenna $\left(N_{D}=1\right)$. In all other scenarios, DH-HSSK and DH-HSM achieve significant energy efficiency gains (in terms of RAER) over DH-W1R. As we see, these gains can be as high as $91 \%$.

\section{F. Power Transmission at the SN and Residual MSE at RNs When I-CSIT Is Available at the SN}

In Section III-F, we demonstrate that the unnormalized MMSE, SRA, and WCRA precoders result in a constrained power transmission, while the ZF precoder does not. Hence, when MMSE, SRA, and WCRA precoding designs are employed in a real system implementation, it is possible to avoid the use of the precoding matrix $\mathbf{D}$ at $\mathrm{SN}$. In this case, given the assumption of $\mathbf{H}_{\mathrm{SR}} \mathbf{P} \approx \mathbf{I}_{N_{R}, N_{R}}$, there is no need for the $i$-th $\mathrm{RN}, i=1, \ldots, N_{R}$ to have the knowledge of $d_{i}$. Thus, we are able to employ a non-coherent detection algorithm at the RNs.
In Fig. 6, using simulation results and the theoretical framework of Section III-G, we evaluate the average precoding power $\bar{P}_{\mathrm{S}}$ at the SN and the residual Mean Square Error (MSE) at each of the RNs, with respect to the variance of channel uncertainty $\sigma_{\widetilde{\mathbf{H}}_{\mathrm{SR}}}^{2}$. This is done for the MMSE, SRA, and WCRA precoding methods when the normalization matrix $\mathbf{D}$ is not applied. We note that due to the fact that $\mathrm{E}_{\mathbf{x}}\left[\|\mathbf{x}\|_{2}^{2}\right]=1$, the instantaneous precoding power $P_{\mathrm{S}}=\|\mathbf{P}\|_{F}^{2}$ dictates the total transmission power.

As shown in Fig. 6, when $\sigma_{\widetilde{\mathbf{H}}_{\mathrm{SR}}}^{2}$ is increased, the power of SRA and WCRA precoding methods is decreased. Thus, as the CSIT at the SN becomes more inaccurate, the power transmission is reduced in order to avoid further degradation (inaccurate beamforming). In contrast, when MMSE precoding is employed and for all the depicted values of $\mathrm{SNR}_{\mathrm{SR}}=\{0,20\}$ $\mathrm{dB}$, the precoding power is increased as the variance of channel uncertainty is increased. This results to more inaccurate beamforming as shown in Fig. 6(b).

Furthermore, Fig. 6 shows that correlation affects the transmitted power of our precoding designs in a composite way. When $\sigma_{\widetilde{\mathbf{H}}_{\mathrm{SR}}}^{2} \rightarrow 0$, higher correlation (higher values of $\rho$ ) results in higher precoding power. In contrast, when $\sigma_{\widetilde{\mathbf{H}}_{\mathrm{SR}}}^{2}$ is increased, higher correlation results in lower precoding power. In contrast to our designs, the existence of channel correlation, when MMSE precoding is used, imposes higher precoding power. As a final remark, we note that all the theoretical curves perfectly match with the simulated curves. In addition, all the claims of Section III-F concerning the relation between the proposed precoding designs and MMSE precoding are confirmed.

Fig. 6(b) demonstrates the residual MSE per RN, defined as $\mathrm{MSE}=\mathrm{E}_{\mathbf{H}_{\mathrm{SR}}}\left[\left\|\mathbf{H}_{\mathrm{SR}} \mathbf{P}-\mathbf{I}_{N_{R}, N_{R}}\right\|_{F}^{2}\right] / N_{R}\left(\mathbf{I}_{N_{R}, N_{R}}\right.$ is the ideal ZF impulse response), which quantifies the residual ICI caused by the inaccurate beamforming, versus the variance of channel uncertainty. As it can be seen, for values of $\sigma_{\widetilde{\mathbf{H}}_{\mathrm{SR}}}^{2}$ close to zero (almost no uncertainty), MMSE results to lower MSE. It is well known that the MMSE precoder is the optimum linear precoder in terms of MSE. Though, as the SN-RNs channel becomes more inaccurate (higher values of $\sigma_{\widetilde{\mathbf{H}}_{\mathrm{SR}}}^{2}$ ) to the SN, our precoding designs offer significantly lower residual MSE than MMSE (for all $\mathrm{SNR}_{\mathrm{SR}}$ values). In the same figure, we depict the performance of the studied precoding designs when the SN-RNs channel is correlated. Clearly, higher correlation results in higher MSE. Finally, we see that as the channel uncertainty becomes high, the effect of correlation to SRA and WCRA is insignificant compared to the uncorrelated case.

\section{G. BER Performance of DH-HSSK/HSM: The Effect of I-CSIT at the $S N$}

In order to acquire a complete picture of the performance of DH-HSSK/HSM under: i) the effect of I-CSIT and ii) different types of precoding at the SN, in Fig. 7, we present the BER curves for both systems when the variance of the SN-RNs channel uncertainty is $\sigma_{\widetilde{\mathbf{H}}_{\mathrm{SR}}}^{2}=\{0.01,0.2\}$. For the sake of comparison, in the same figures we include the performance of DH-HSSK/HSM when MMSE precoding with P-CSIT is 


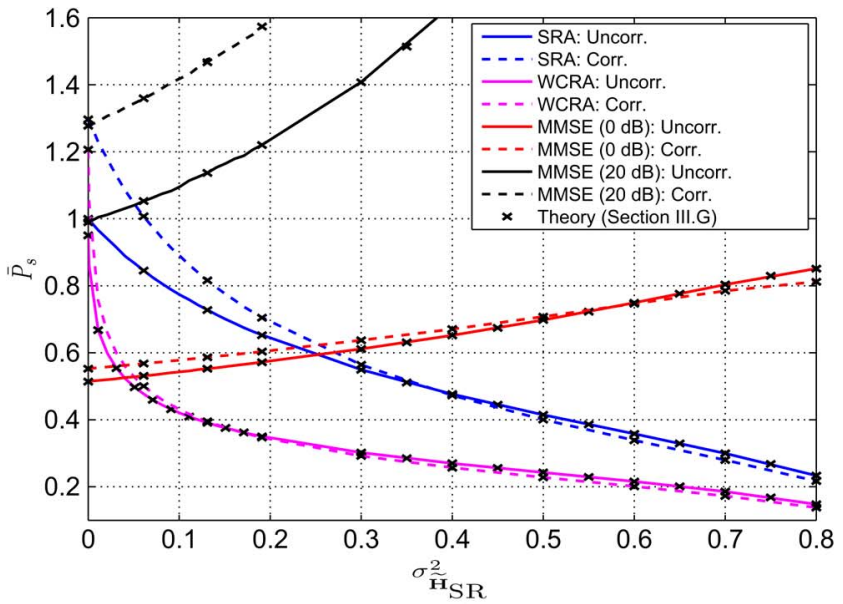

(a)

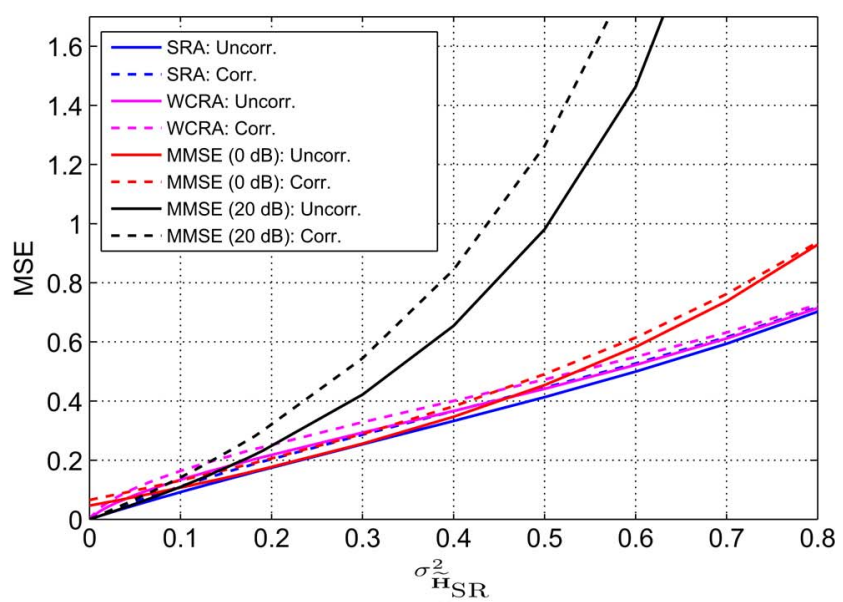

(b)

Fig. 6. Effect of I-CSIT at the SN of a $8 \times 4 \times 4$ system. (a) Average precoding power at the SN. (b) Residual MSE at each RN.

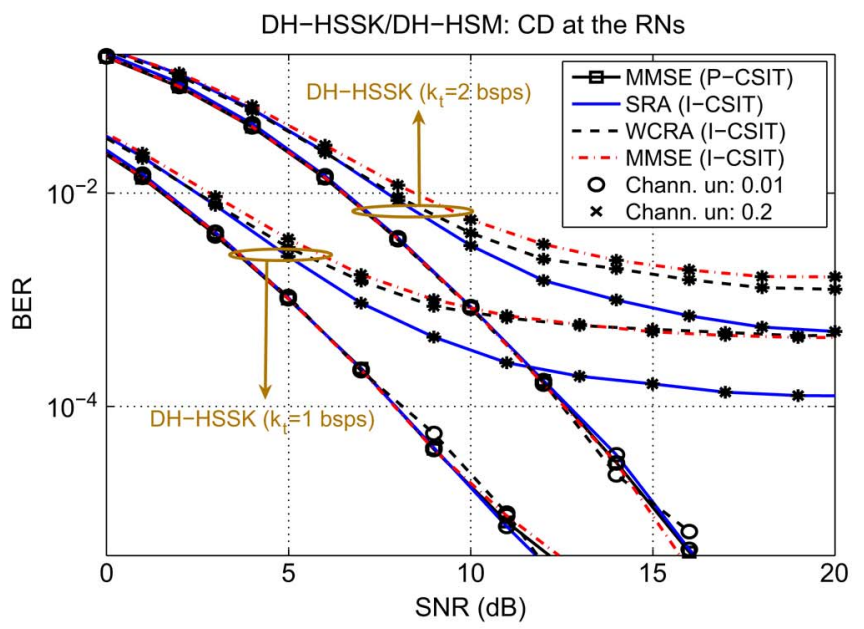

(a)

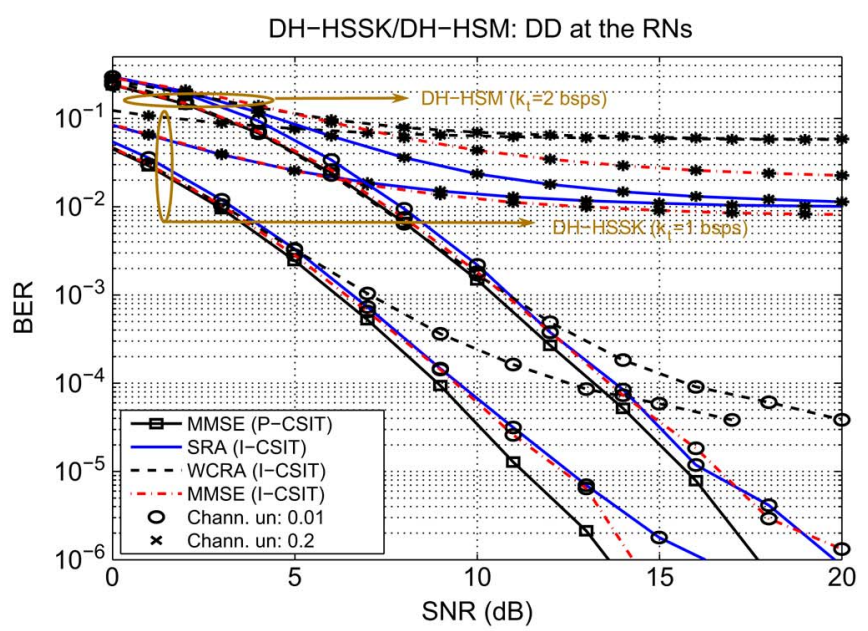

(b)

Fig. 7. BER performance of a $8 \times 4 \times 4$ DH-HSSK $\left(k_{t}=1\right.$ bpsp $)$ and a $8 \times 4 \times 4$ DH-HSM $\left(k_{t}=2\right.$ bpsp $)$ system, when different values of channel uncertainty for the SN-RNs channel $\left(\sigma_{\widetilde{\mathbf{H}}_{\mathrm{SR}}^{2}}^{2}=\{0.01,0.2\}\right)$ are used. (a) CD at the RNs. (b) DD at the RNs.

employed at the SN. In addition, we assume that the SN-RNs and RNs-DN channels are uncorrelated. ${ }^{2}$

As it can be seen from Fig. 7(a), when CD is employed at the RNs and the channel uncertainty is small $\left(\sigma_{\widetilde{\mathbf{H}}_{\mathrm{SR}}}^{2}=0.01\right)$, DH-HSSK and DH-HSM have no performance difference from the ideal scenario of P-CSIT at the SN. This is true for all types of precoding. In contrast, the increase of channel uncertainty to $\sigma_{\widetilde{\mathbf{H}}_{\mathrm{SR}}}^{2}=0.2$ causes a BER performance degradation. In low $\mathrm{SNR}$, the degradation is about $1 \mathrm{~dB}$. Though, an error floor is caused in high SNR, due to the noise limited detection at the RNs. In fact, this is the effect of inaccurate beamforming from the SN. Finally, from Fig. 7(a), we conclude that when I-CSIT is available at the $\mathrm{SN}$, the optimal precoding method is SRA.

In addition, Fig. 7(b) demonstrates that the effect of I-CSIT has a more diminishing result for the case of DD. In fact, in low SNR, there is a small BER performance loss even for low

\footnotetext{
${ }^{2}$ We note that, in order to provide a fair comparison between the different precoding methods, we make use of the normalization matrix $\mathbf{D}$ as defined in Section II
}

channel uncertainty $\left(\sigma_{\widetilde{\mathbf{H}}_{\mathrm{SR}}}^{2}=0.01\right)$, both for DH-HSSK and DH-HSM. Moreover, after $10 \mathrm{~dB}$, the phenomenon of the error floor in high SNR starts to appear. Finally, we see that high channel uncertainty $\left(\sigma_{\widetilde{\mathbf{H}}_{\mathrm{SR}}}^{2}=0.2\right)$ causes a diminishing BER performance, even in high SNRs.

In Fig. 8, we demonstrate the BER performance of DHHSSK/DH-HSM under the composite effect of: i) I-CSIT at the $\mathrm{SN}$ and ii) correlated $\mathrm{SN}-\mathrm{RNs}$ and RNs-DN channel. The variance of channel uncertainty at the $\mathrm{SN}$ is $\sigma_{\widetilde{\mathbf{H}}_{\mathrm{SR}}}^{2}=0.05$. Furthermore, the correlation scenarios that we consider are: i) low correlation with $\rho=0.1$ and ii) high correlation with $\rho=0.5$. In addition, given that the RNs are placed far apart, the SN-RNs channel contains only transmit space correlation, while the RNs-DN channel is affected only by receive space correlation. As a benchmark reference, we employ the ideal scenario of P-CSIT at the SN and uncorrelated SN-RNs and RNs-DN channels.

Fig. 8(a) shows that for the depicted SNRs and when correlation is low $\rho=0.1$, the performance of the proposed architecture with $\mathrm{CD}$ does not deviate from the ideal case of no 


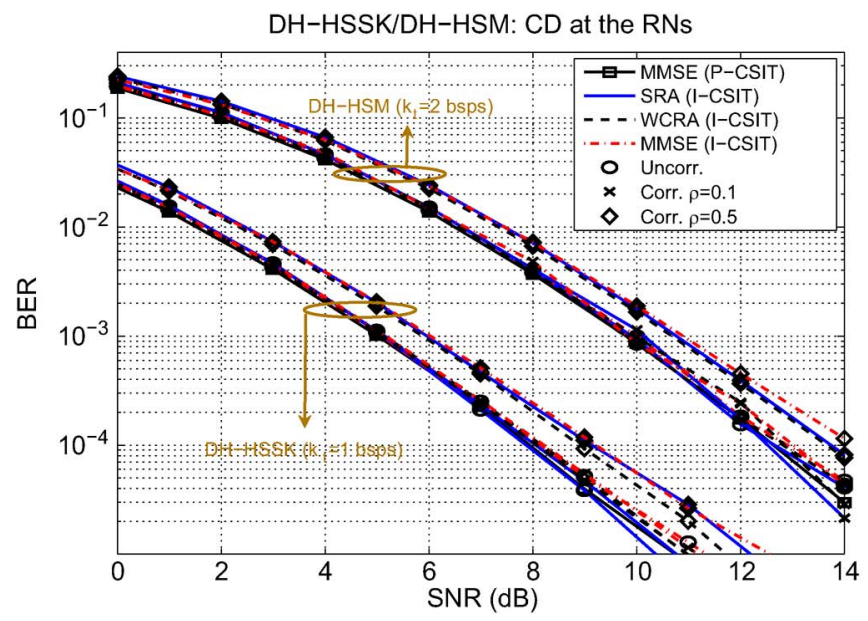

(a)

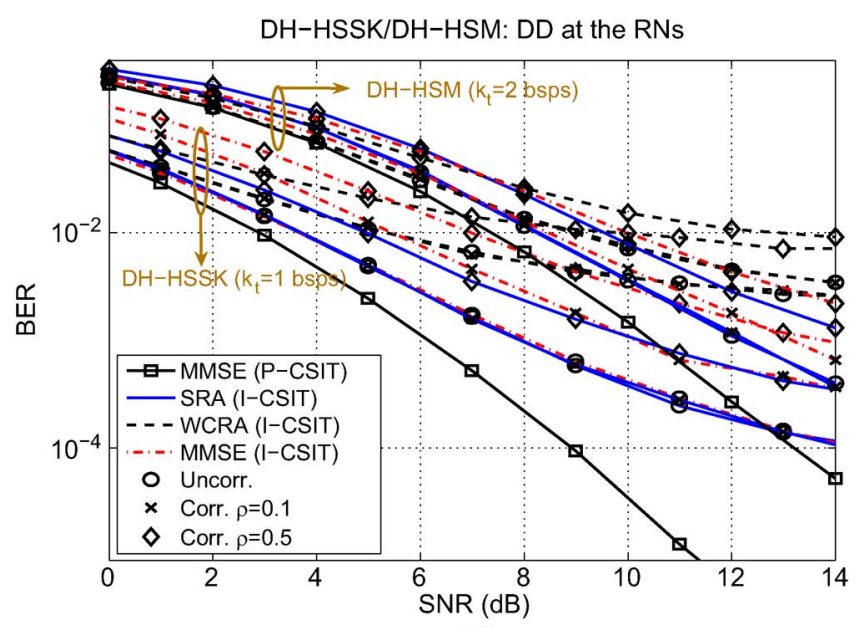

(b)

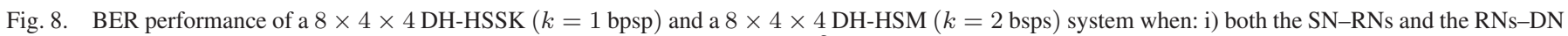
channels are correlated with $\rho=\{0.1,0.5\}$ and ii) the SN possesses I-CSIT with $\sigma_{\widetilde{H}_{\mathrm{SR}}}^{2}=0.05$. (a) CD at the RNs. (b) DD at the RNs.

correlation and P-CSIT at the SN. Furthermore the inspection of the same figure shows that high correlation degrades the BER performance for about $1 \mathrm{~dB}$ at $\mathrm{BER}=10^{-3}$. In fact, the previous conclusions are practically true for all methods of precoding. Moreover, Fig. 8(b) presents that the use of DD at the RNs results in a diminishing behavior, even for low correlation. Finally, Fig. 8(b) demonstrates that SRA is the best precoding method under the evaluated system setup.

\section{CONCLUSION}

This paper focused on the extension of the concept of space modulation to a VMIMO architecture. Based on the ideas of R-SM and SM, we formed an architecture which transfers information using the spatial position of multiple RNs, in addition, or not, to the conventional way. Using MIMO linear precoding, we were able to reduce or practically avoid the native problem of the activation of multiple RNs of the space modulated VMIMO schemes.

We compared our architecture against the appropriate SotA schemes and demonstrated significant BER gains. Especially, for a very large system setup and $\mathrm{BER}=10^{-4}$, the performance difference was up to $9 \mathrm{~dB}$ for $\mathrm{DH}$-wBRS and up to $10 \mathrm{~dB}$ for Dh-w1R. Hence, we concluded that the concept of space modulation can be successfully applied in a distributed framework. Moreover, we provided strict bounds for the ABEP, both for the $\mathrm{CD}$ and DD algorithms at the RNs.

In order to provide linear precoding using a practical scenario of I-CSIT, we proposed two precoding methods. The first precoder is based on a statistical approach, while, the second precoder follows a worst case method. The instantaneous and average power of each precoder, used in this paper, was analyzed using a theoretical framework. The theoretical and simulated curves perfectly match. Using simulation results, we came to the conclusion that our architecture and precoding methods are robust to realistic scenarios of CSIT and correlation.

\section{REFERENCES}

[1] E. Telatar, "Capacity of multi-antenna Gaussian channels," Eur. Trans. Telecommun., vol. 10, no. 6, pp. 585-595, Nov./Dec. 1999.

[2] E. Larsson, "MIMO detection methods: How they work," IEEE Signal Process. Mag., vol. 26, no. 3, pp. 91-95, May 2009.

[3] A. Stavridis, S. Sinanović, M. D. Renzo, and H. Haas, "Energy evaluation of spatial modulation at a multi-antenna base station," in Proc. 78th IEEE VTC, Las Vegas, NV, USA, Sep. 2-5, 2013, pp. 1-5.

[4] A. del Coso, U. Spagnolini, and C. Ibars, "Cooperative distributed MIMO channels in wireless sensor networks," IEEE J. Sel. Areas Commun., vol. 25 , no. 2, pp. 402-414, Feb. 2007.

[5] S. Jayaweera, "V-BLAST-based virtual MIMO for distributed wireless sensor networks," IEEE Trans. Commun., vol. 55, no. 10, pp. 1867-1872, Oct. 2007.

[6] H. Chen, A. Gershman, and S. Shahbazpanahi, "Filter-and-forward distributed beamforming in relay networks with frequency selective fading," IEEE Trans. Signal Process., vol. 58, no. 3, pp. 1251-1262, Mar. 2010.

[7] L. Lai, K. Liu, and H. El-Gamal, "The three-node wireless network: Achievable rates and cooperation strategies," IEEE Trans. Inf. Theory, vol. 52, no. 3, pp. 805-828, Mar. 2006.

[8] G. Kramer, M. Gastpar, and P. Gupta, "Cooperative strategies and capacity theorems for relay networks," IEEE Trans. Inf. Theory, vol. 51, no. 9, pp. 3037-3063, Sep. 2005.

[9] X. Hong, C.-X. Wang, M. Uysal, X. Ge, and S. Ouyang, "Capacity of hybrid cognitive radio networks with distributed VAAs," IEEE Trans. Veh. Technol., vol. 59, no. 7, pp. 3510-3523, Sep. 2010.

[10] R. Mesleh, H. Haas, S. Sinanović, C. W. Ahn, and S. Yun, "Spatial modulation," IEEE Trans. Veh. Technol., vol. 57, no. 4, pp. 2228-2241, Jul. 2008.

[11] J. Jeganathan, A. Ghrayeb, and L. Szczecinski, "Spatial modulation: Optimal detection and performance analysis," IEEE Commun. Lett., vol. 12, no. 8 , pp. 545-547, Aug. 2008.

[12] M. Di Renzo, H. Haas, and P. M. Grant, "Spatial modulation for multipleantenna wireless systems: A survey," IEEE Commun. Mag., vol. 49, no. 12, pp. 182-191, Dec. 2011.

[13] J. Jeganathan, A. Ghrayeb, L. Szczecinski, and A. Ceron, "Space shift keying modulation for MIMO channels," IEEE Trans. Wireless Commun., vol. 8, no. 7, pp. 3692-3703, Jul. 2009.

[14] N. Serafimovski et al., "Practical implementation of spatial modulation," IEEE Trans. Veh. Technol., vol. 62, no. 9, pp. 4511-4523, Nov. 2013.

[15] A. Younis et al., "Performance of spatial modulation using measured real-world channels," in Proc. 78th IEEE VTC, Las Vegas, NV, USA, Sep. 2-5, 2013, pp. 1-5.

[16] J. Zhang, Y. Wang, L. Ding, and N. Zhang, "Bit error probability of spatial modulation over measured indoor channels," IEEE Trans. Wireless Commun., vol. 13, no. 3, pp. 1380-1387, Mar. 2014.

[17] M. Di Renzo, H. Haas, A. Ghrayeb, S. Sugiura, and L. Hanzo, "Spatial modulation for generalized MIMO: Challenges, opportunities, implementation," Proc. IEEE, vol. 102, no. 1, pp. 56-103, Jan. 2014. 
[18] L.-L. Yang, "Transmitter preprocessing aided spatial modulation for multiple-input-multiple-output systems," in Proc. 73rd IEEE VTC Spring, May 2011, pp. 1-5.

[19] A. Stavridis, S. Sinanović, M. Di Renzo, and H. Haas, "Transmit precoding for receive spatial modulation using imperfect channel knowledge," in Proc. 75th IEEE VTC Spring, 2012, pp. 1-5.

[20] R. Mesleh, S. Ikki, and M. Alwakeel, "Performance analysis of space shift keying with amplify and forward relaying," IEEE Commun. Lett., vol. 15, no. 12, pp. 1350-1352, Dec. 2011.

[21] M. Raed, I. Salama, A. Hadi, and A. Mansour, "Performance analysis of Space Shift Keying (SSK) modulation with multiple cooperative relays," EURASIP J. Adv. Signal Process., vol. 2012, no. 1, p. 201, Sep. 2012.

[22] M. Wen, X. Cheng, V. Poor, and B. Jiao, "Use of Space Shift Keying (SSK) modulation in two-way amplify-and-forward relaying," IEEE Trans. Veh. Technol., vol. 63, no. 3, pp. 1498-1504, Mar. 2014.

[23] P. Som and A. Chockalingam, "End-to-end BER analysis of space shift keying in decode-and-forward cooperative relaying," in Proc. IEEE WCNC, 2013, pp. 3465-3470.

[24] P. Som and A. Chockalingam, "BER analysis of space shift keying in cooperative multi-hop multi-branch DF relaying," in Proc. 78th IEEE VTC, Las Vegas, NV, USA, Sep. 2-5, 2013, pp. 1-5.

[25] S. Sugiura, S. Chen, and L. Hanzo, "Generalized space-time shift keying designed for flexible diversity-, multiplexing- and complexitytradeoffs," IEEE Trans. Wireless Commun., vol. 10, no. 4, pp. 1144-1153, Apr. 2011.

[26] S. Sugiura, S. Chen, H. Haas, P. Grant, and L. Hanzo, "Coherent versus non-coherent decode-and-forward relaying aided cooperative space-time shift keying," IEEE Trans. Commun., vol. 59, no. 6, pp. 1707-1719, Jun. 2011.

[27] N. Serafimovski, S. Sinanovic, M. Di Renzo, and H. Haas, "Dual-hop Spatial Modulation (Dh-SM)," in Proc. IEEE VTC Spring, Budapest, Hungary, May 15-18, 2011, pp. 1-5.

[28] S. Narayanan, M. Di Renzo, F. Graziosi, and H. Haas, "Distributed spatial modulation for relay networks," in Proc. 78th IEEE VTC, Las Vegas, NV, USA, Sep. 2-5, 2013, pp. 1-6.

[29] S. Narayanan, M. Di Renzo, F. Graziosi, and H. Haas, "Distributed space shift keying for the uplink of relay-aided cellular networks," in Proc. IEEE 17th Int. Workshop CAMAD Commun. Links Netw., 2012, pp. $130-134$

[30] Y. Yang and S. Aissa, "Information-guided transmission in decode-andforward relaying systems: Spatial exploitation and throughput enhancement," IEEE Trans. Wireless Commun., vol. 10, no. 7, pp. 2341-2351, Jul. 2011.

[31] P. Yang et al., "Detect-and-forward relaying aided cooperative spatial modulation for wireless networks," IEEE Trans. Commun., vol. 61, no. 11, pp. 4500-4511, Nov. 2013.

[32] S. Narayanan, A. Stavridis, M. Di Renzo, F. Graziosi, and H. Haas, "Distributed spatially-modulated space-time-block-codes," in Proc. IEEE 18th Int. Workshop CAMAD Commun. Links Netw., 2013, pp. 159-163.

[33] A. Stavridis, S. Sinanović, M. D. Renzo, and H. Haas, "A power saving dual-hop architecture based on hybrid spatial modulation," in Conf. Rec. 46th ASILOMAR Conf. Signals, Syst. Comput., Nov. 4-7, 2012, pp. 1366-1370.

[34] J. G. Proakis and M. Salehi, Communication System Engineering. Upper Saddle River, NJ, USA: Prentice-Hall, 1994.

[35] A. Wiesel, Y. Eldar, and S. Shamai, "Zero-forcing precoding and generalized inverses," IEEE Trans. Signal Process., vol. 56, no. 9, pp. 4409-4418, Sep. 2008

[36] C. Peel, B. Hochwald, and A. Swindlehurst, "A vector-perturbation technique for near-capacity multiantenna multiuser communication-Part I: Channel inversion and regularization," IEEE Trans. Commun., vol. 53, no. 1, pp. 195-202, Jan. 2005.

[37] W. Ho, T. Quek, S. Sun, and R. Heath, "Decentralized precoding for multicell MIMO downlink," IEEE Trans. Wireless Commun., vol. 10, no. 6, pp. 1798-1809, Jun. 2011.

[38] S. Boyd and L. Vandenberghe, Convex Optimization. Cambridge, U.K.: Cambridge Univ., 2004.

[39] A. Tulino, A. Lozano, and S. Verdu, "Impact of antenna correlation on the capacity of multiantenna channels," IEEE Trans. Inf. Theory, vol. 51, no. 7, pp. 2491-2509, Jul. 2005.

[40] A. M. Tulino and S. Verdu, "Random matrix theory and wireless communications," Found. Trends Commun. Inf. Theory, vol. 1, no. 1, pp. 1-182, Jun. 2004.

[41] I. S. Gradshteyn and I. M. Ryzhik, Table of Integrals, Series, Products, A. Jeffrey and D. Zwillinger, Eds., 7th ed. San Diego, CA, USA: Academic, Mar. 2007.
[42] G. Alfano, A. Tulino, A. Lozano, and S. Verdu, "Capacity of MIMO channels with one-sided correlation," in Proc. IEEE 8th Int. Symp. Spread Spectr. Tech. Appl., Aug. 2004, pp. 515-519.

[43] A. Mehana and A. Nosratinia, "Diversity of MIMO linear precoding," IEEE Trans. Inf. Theory, vol. 60, no. 2, pp. 1019-1038, Feb. 2014

[44] N. Jindal and A. Goldsmith, "Dirty-paper coding versus TDMA for MIMO broadcast channels," IEEE Trans. Inf. Theory, vol. 51, no. 5, pp. 1783-1794, May 2005.

[45] M. O. Hasna and M.-S. Alouini, "End-to-end performance of transmission systems with relays over Rayleigh-fading channels," IEEE Trans. Wireless Commun., vol. 2, no. 6, pp. 1126-1131, Nov. 2003.

[46] A. Younis, D. A. Basnayaka, and H. Haas, "Performance analysis for generalised spatial modulation," in Proc. EW Conf., Barcelona, Spain, May 14-16, 2014, pp. 207-212.

[47] M. Di Renzo and H. Haas, "Bit error probability of space modulation over Nakagami- $m$ fading: Asymptotic analysis," IEEE Commun. Lett., vol. 15, no. 10, pp. 1026-1028, Oct. 2011.

[48] D. Gore, R. Heath, and A. Paulraj, "Transmit selection in spatial multiplexing systems," IEEE Commun. Lett., vol. 6, no. 11, pp. 491-493, Nov. 2002.

[49] N. Balakrishnan and C. D. Lai, Continuous Bivariate Distributions. New York, NY, USA: Springer-Verlag, 2009.

[50] N. Serafimovski, S. Sinanovic, M. Renzo, and H. Haas, "Multiple access spatial modulation," EURASIP J. Wireless Commun. Netw., vol. 2012, no. 1, pp. 299-1-299-20, Sep. 2012.

[51] F. Rusek et al., "Scaling up MIMO: Opportunities and challenges with very large arrays," IEEE Signal Process. Mag., vol. 30, no. 1, pp. 40-60, Jan. 2013.

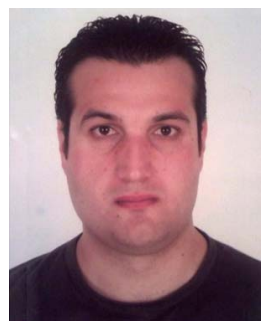

Athanasios Stavridis (S'12) was born in Kastoria, Greece. He holds the Diploma degree (M.Eng. equivalent) in computer engineering and informatics and the M.Sc. degree in signal processing and communication systems from the University of Patras, Patras, Greece, in 2006 and 2008, respectively. He is currently working toward the Ph.D. degree with The University of Edinburgh, Edinburgh, U.K. He was awarded with a scholarship from the University of Patras upon the completion of his M.Sc. studies.

During the period of June 2011-June 2014, he was a Marie Curie Early-Stage-Researcher at The University of Edinburgh, Edinburgh, U.K. In January 2014, he was selected as an Exemplary Reviewer of the IEEE Communication Letters Reviewer Appreciation Program 2013. His main research interests include energy efficient physical layer techniques for wireless communications, spatial modulation, MIMO systems, and cooperative communications.

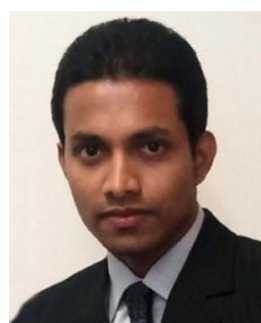

Dushyantha Basnayaka (S'11-M'12) received the B.Sc.Eng. degree (with first class honors) from the University of Peradeniya, Peradeniya, Sri Lanka, in 2006 and the Ph.D. degree in electrical engineering from the University of Canterbury, Christchurch, New Zealand, in 2012.

He was a System Engineer at MillenniumIT (a member company of the London Stock Exchange Group) from 2006 to 2009. He was with the Communication Research Group, University of Canterbury, from 2009 until 2012. He is now a Research Fellow in Wireless Communication at The University of Edinburgh, Edinburgh, U.K. $\mathrm{He}$ is the holder of one U.S. patent. His current research interests include massive MIMO, spatial modulation, interference mitigation techniques for cellular wireless systems, and macrodiversity multiuser MIMO systems.

Dr. Basnayaka was a recipient of University of Canterbury International Doctoral Scholarship for his doctoral studies at the University of Canterbury. 


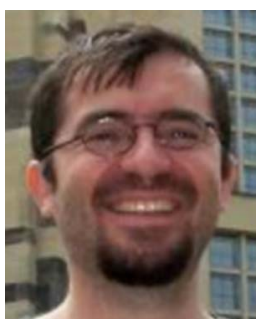

Sinan Sinanovic (S'98-M'07) received the Ph.D. degree in electrical and computer engineering from Rice University, Houston, TX, USA, in 2006. In 2006, he joined Jacobs University Bremen, Germany, as a Postdoctoral Fellow. In 2007, he joined The University of Edinburgh, U.K., where he was a Research Fellow at the Institute for Digital Communications. While working with Halliburton Energy Services, he has developed acoustic telemetry receiver, which was patented. He has also worked for Texas Instruments on development of ASIC testing. He is currently a Lecturer at Glasgow Caledonian University, Glasgow, U.K. Dr. Sinanovic is a member of the Tau Beta Pi Engineering Honor Society and a member of Eta Kappa Nu Electrical Engineering Honor Society. He won an honorable mention at the International Math Olympiad in 1994.

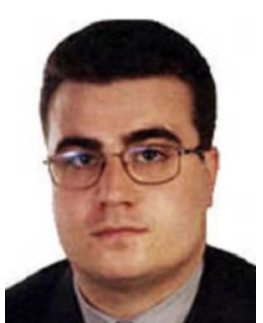

Marco Di Renzo (S'05-A'07-M'09-SM'14) received the Laurea (cum laude) and Ph.D. degrees in electrical and information engineering from the University of L'Aquila, L'Aquila, Italy, in 2003 and 2007, respectively, and the Habilitation à Diriger des Recherches from the University of Paris-Sud XI, Paris, France, in 2013.

Since January 2010, he has been a Tenured Academic Researcher ("Chargé de Recherche Titulaire") with the French National Center for Scientific Research (CNRS), as well as a Faculty Member of the Laboratory of Signals and Systems (L2S), a joint research laboratory of the CNRS, the École Supérieure d'Électricité (SUPÉLEC), and the University of Paris-Sud XI. His main research interests are in the area of wireless communications theory.

Dr. Di Renzo currently serves as an Editor of the IEEE COMMUNICATIONS LETTERS and the IEEE TRANSACTIONS ON COMMUNICATIONS (Heterogeneous Networks Modeling and Analysis). He was a recipient of several awards, which include a special mention for the outstanding five-year (1997-2003) academic career, University of L'Aquila; the THALES Communications Fellowship (2003-2006), University of L'Aquila; the 2004 Best SpinOff Company Award, Abruzzo Region, Italy; the 2006 DEWS Travel Grant Award, University of L'Aquila; the 2008 Torres Quevedo Award, Ministry of Science and Innovation, Spain; the "Dérogation pour l'Encadrement de Thèse" (2010), University of Paris-Sud XI; the 2012 IEEE CAMAD Best Paper Award; the 2012 IEEE WiRELESS COMMUNICATIONS LETTERS Exemplary Reviewer Award; the 2013 IEEE VTC-Fall Best Student Paper Award; the 2013 Network of Excellence NEWCOM\# Best Paper Award; the 2013 IEEE Transactions on Vehicular Technology Top Reviewer Award; the 2013 IEEE-COMSOC Best Young Researcher Award for Europe, Middle East and Africa (EMEA Region); and the 2014 IEEE ICNC Single Best Paper Award Nomination (Wireless Communications Symposium).

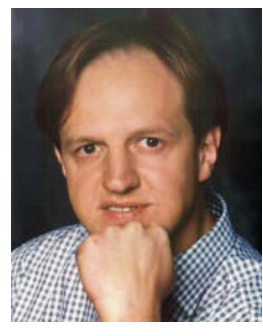

Harald Haas received the Ph.D. degree from The University of Edinburgh, Edinburgh, U.K., in 2001. He holds the Chair of Mobile Communications at The University of Edinburgh, Edinburgh, U.K. He has published 280 conference and journal papers, including a paper in Science. He holds 26 patents and has more than 20 pending patent applications. His main research interests are in optical wireless communications, hybrid optical wireless and RF communications, spatial modulation and massive MIMO, and interference coordination in wireless networks. Prof. Haas was an invited speaker at TED Global 2011, and his talk has been watched online more than 1.5 million times. He is a Co-Founder and a Chief Scientific Officer of pureLiFi Ltd. He first introduced and coined "spatial modulation" and "Li-Fi". Li-Fi was listed among the 50 best inventions in TIME Magazine 2011. He was co-recipient of a Best Paper Award at the IEEE Vehicular Technology Conference in Las Vegas in 2013. In 2012, he was the only recipient of the prestigious Established Career Fellowship from the Engineering and Physical Sciences Research Council (EPSRC) within Information and Communications Technology in the U.K. He was a recipient of the Tam Dalyell Prize 2013 awarded by The University of Edinburgh for excellence in engaging the public with science. In 2014, he was selected by EPSRC as one of ten RISE (Recognising Inspirational Scientists and Engineers) Leaders. 